\title{
AN EIGENSPACE OF LARGE DIMENSION FOR A HECKE ALGEBRA ON AN $\tilde{A}_{2}$ BUILDING
}

\author{
A. M. MANTERO and A. ZAPPA ${ }^{\bowtie}$ \\ (Received 3 June 2010; accepted 1 October 2010) \\ Communicated by M. G. Cowling
}

\begin{abstract}
Let $\Delta$ be an affine building of type $\tilde{A}_{2}$ and let $\mathbb{A}$ be its fundamental apartment. We consider the set $\mathbb{U}_{0}$ of vertices of type 0 of $\mathbb{A}$ and prove that the Hecke algebra of all $W_{0}$-invariant difference operators with constant coefficients acting on $\mathbb{U}_{0}$ has three generators. This property leads us to define three Laplace operators on vertices of type 0 of $\Delta$. We prove that there exists a joint eigenspace of these operators having dimension greater than $\left|W_{0}\right|$. This implies that there exist joint eigenfunctions of the Laplacians that cannot be expressed, via the Poisson transform, in terms of a finitely additive measure on the maximal boundary $\Omega$ of $\Delta$.
\end{abstract}

2010 Mathematics subject classification: primary 51E24; secondary 20C08, 43A80.

Keywords and phrases: buildings, Hecke algebras, Laplace operators, Poisson kernel.

\section{Introduction}

Let $\Delta$ be an affine building of rank two. We denote by $\mathbb{A}$ its fundamental apartment and by $W_{0}$ its finite Weyl group. The set $\mathbb{U}$ of all special vertices of $\mathbb{A}$ is a lattice of the Euclidean space $\mathbb{R}^{2}$ and we denote by $\mathcal{H}(\mathbb{A})$ the Hecke algebra of all $W_{0}$-invariant difference operators with constant coefficients acting on the set of all complex-valued functions defined on $\mathbb{U}$. We consider the algebra $\mathcal{H}(\Delta)$, obtained as the linear span of all averaging operators $A_{m, n}$, where $m, n \in \mathbb{Z}$, defined by

$$
A_{m, n} f(x)=\left|S_{m, n}(x)\right|^{-1} \sum_{y \in S_{m, n}(x)} f(y),
$$

for every complex-valued function $f$ on the set $\mathcal{U}$ of all special vertices of the building. Here, $S_{m, n}(x)$ denotes the set of all special vertices having coordinates $(m, n)$ with respect to $x$. Both the algebras $\mathcal{H}(\mathbb{A})$ and $\mathcal{H}(\Delta)$ have two generators, called Laplace operators on $\mathbb{A}$ and on $\Delta$ respectively.

In [6-8], we used a case-by-case analysis to generalize a classical result of Helgason [2] on rank-one symmetric spaces. For buildings of type $\tilde{A}_{2}, \tilde{B}_{2}$ and $\tilde{G}_{2}$,

(c) 2011 Australian Mathematical Publishing Association Inc. 1446-7887/2011 \$16.00 
our results characterize the joint eigenfunctions of the Laplacians on the building as Poisson transforms of finitely additive measures on the maximal boundary $\Omega$ of $\Delta$.

The definition of $W_{0}$-invariant operators on a subset of $\mathbb{U}$ requires this set to be a sublattice of $\mathbb{U}$. We note that the only sublattice of $\mathbb{U}$ containing the fundamental vertex $O=(0,0)$ is the set of all vertices of the same type as $O$, say type 0 . In this case, we can replace $\mathcal{H}(\mathbb{A})$ and $\mathcal{H}(\Delta)$ by algebras, $\mathcal{H}_{0}(\mathbb{A})$ and $\mathcal{H}_{0}(\Delta)$, associated with the vertices of type 0 . It is trivial to see that $\mathcal{H}(\mathbb{A})=\mathcal{H}_{0}(\mathbb{A})$ and that $\mathcal{H}(\Delta)=\mathcal{H}_{0}(\Delta)$ for buildings of type $\tilde{G}_{2}$.

The algebras $\mathcal{H}_{0}(\mathbb{A})$ and $\mathcal{H}_{0}(\Delta)$ were considered in [7] for a building of type $\tilde{B}_{2}$. They have two generators, called Laplace operators for the type 0 vertices. In this case, we proved that the generalization of the classical result of Helgason, that is, any joint eigenfunction of all differential operators on a symmetric space can be given by the Poisson integral, fails for some choice of both the eigenvalues and the parameter system for the building.

In this paper, we consider the algebras $\mathcal{H}_{0}(\mathbb{A})$ and $\mathcal{H}_{0}(\Delta)$ for a building of type $\tilde{A}_{2}$. This case differs from those of other buildings of rank two because $\mathcal{H}_{0}(\mathbb{A})$ has three generators, and so there are three Laplace operators on the vertices of type 0 of $\mathbb{A}$ and $\Delta$ respectively. This property leads us to prove that there exists a particular choice of the eigenvalues for the Laplacians such that the joint eigenspace has dimension greater than the cardinality of the finite Weyl group $W_{0}$ for the building.

It is interesting to remark that this is the only case, among buildings of rank two, in which there exist eigenvalues for the Laplacians having this property. Actually, in all other cases it has been proved that the dimension of every eigenspace of the Laplacians is always $\left|W_{0}\right|$. See [6-8].

Since each triple of eigenvalues for the Laplacians generates an eigenvalue of the Hecke algebra and arises from a multiplicative function defined on $\mathbb{U}_{0}$, the multiplicative function associated with the singular triple of eigenvalues gives an example of a character whose stabilizer in $W_{0}$ is not a reflection group (see [3, Proposition 1.1]). We apply this information about the features of the joint eigenspaces of the Laplacians to generalize Helgason's result to the context of these operators. We find that, whatever the parameter system of the building may be, this generalization fails for the triple of singular eigenvalues. This is because there are joint eigenfunctions of the Laplace operators, associated with this triple, that cannot be expressed, via the Poisson transform, in terms of a finitely additive measure on the maximal boundary of $\Delta$.

This paper concludes the study of the generalization of Helgason's result for buildings of rank two, begun in [6] for all vertices of a type $\tilde{A}_{2}$ building, and continued in $[7,8]$ for buildings of type $\tilde{B}_{2}$ and $\tilde{G}_{2}$. The strategy that we use in resolving the problem is the same as that for the other types of buildings of rank two, and we refer the reader to [6-8] for its description.

In Section 2, we fix a coordinate system on $\mathbb{U}_{0}$, and compare it with the coordinate system defined in [6] for all vertices of $\mathbb{A}$. In Section 3 we consider the Poisson kernel defined on $\mathcal{U}_{0}$, and define matrices $\mathbb{M}_{0}, \mathbb{M}_{1}, \mathbb{M}_{2}$ that allow us to compute the retraction of the Poisson kernel, with respect to a chamber, according to the method described in $[7,8]$. 
In Sections 4 and 5 we investigate the Hecke algebra $\mathcal{H}_{0}(\mathbb{A})$. We prove that the algebra has three generators, and exhibit the relations between them. Moreover, we define Laplace operators on the set $\mathcal{U}_{0}$, and determine their retractions on the fundamental apartment.

Sections 6 and 7 are devoted to the study of the joint eigenfunctions of the Laplace operators on $\mathbb{A}$ and on $\Delta$ respectively. In particular, in Section 6 , we determine the dimensions of the joint eigenspaces of these operators, and prove that, for a particular choice of eigenvalue, the joint eigenspace has dimension greater than the cardinality of the finite Weyl group $W_{0}$. Finally, in Section 8 , we investigate the bijectivity of the Poisson transform, and we show that in one case the generalization of Helgason's result fails.

We direct the interest reader to the article of Kellil and Rousseau [4] for information on related arguments.

\section{Coordinates on the fundamental apartment}

We refer to [6, 9] for formal definitions and details about the features of buildings of type $\tilde{A}_{2}$. We let $q$ denote the homogeneity of the building. We let $\mathcal{U}_{0}$ and $\mathbb{U}_{0}$ denote the set of all type 0 vertices of $\Delta$ and of the fundamental apartment $\mathbb{A}$ respectively. We fix a sector $\mathbb{Q}_{0}$ on $\mathbb{A}$ based at a vertex $O \in \mathbb{U}_{0}$. We denote its fundamental chamber by $C_{0}$.

In [6] we defined a pair of integer coordinates for all vertices of any apartment $\mathcal{A}$, with respect to a fixed sector $Q_{x_{0}}$ of the apartment. It is easy to check that, if we choose $x_{0} \in \mathcal{U}_{0}$, then a vertex $x$ has type 0 if and only if it has coordinates $(m, n)$ such that

$$
(m, n)=(3 l+h, h)
$$

for some $h, l \in \mathbb{Z}$, as shown in Figure 1. In particular, these coordinates can be defined on the fundamental apartment $\mathbb{A}$, with respect to $\mathbb{Q}_{0}$. We note that $x$ belongs to the sector $Q_{x_{0}}$ if and only if $h \geq 0$, whereas $l$ may be negative.

Later on, it will be useful to consider a different coordinate system for the vertices of $\mathcal{U}_{0}$ lying on an apartment. As illustrated in Figure 2, let $Q_{x_{0}}^{\prime}$ be the sector 2-adjacent to $Q_{x_{0}}$. Then, let $H_{1}$ and $H_{2}$ denote the bisector lines of $Q_{x_{0}}^{\prime}$ and $Q_{x_{0}}$ respectively, oriented toward $Q_{x_{0}}^{\prime}$ and $Q_{x_{0}}$. We assign coordinates $\left(m_{0}, 0\right)$, where $m_{0} \in \mathbb{Z}$, to the type 0 vertex of $H_{1}$ at distance $2\left|m_{0}\right|$ from $x_{0}$ and coordinates $\left(0, n_{0}\right)$, where $n_{0} \in \mathbb{Z}$, to the type 0 vertex of $H_{2}$ at distance $2\left|n_{0}\right|$ from $x_{0}$, according to the orientation of $H_{1}$ and $H_{2}$.

As usual, these coordinates are independent of the apartment containing the vertex and the sector. In particular, vertices lying on two different sectors, based at the same vertex of $\mathcal{U}_{0}$, have the same coordinates with respect to both sectors. For any $x \in \mathcal{A}$ of type 0 , let $(m, n)$ and $\left(m_{0}, n_{0}\right)$ denote its coordinates in $\mathcal{U}$ and in $\mathcal{U}_{0}$ respectively, with respect to a sector $Q_{x_{0}}$ based at a vertex of type 0 . Then

$$
m=2 m_{0}+n_{0} \quad \text { and } \quad n=n_{0}-m_{0} .
$$

In particular, the vertices of $\mathcal{U}_{0}$ lying on the sector $Q_{x_{0}}$ are characterized by coordinates $\left(m_{0}, n_{0}\right)$ such that $-n_{0} / 2 \leq m_{0} \leq n_{0}$. From now on, we set $X=X_{m, n}$ or $X=X_{m_{0}, n_{0}}$ if the vertex $X$ has coordinates $(m, n)$ or $\left(m_{0}, n_{0}\right)$ with respect to $\mathbb{Q}_{0}$. 


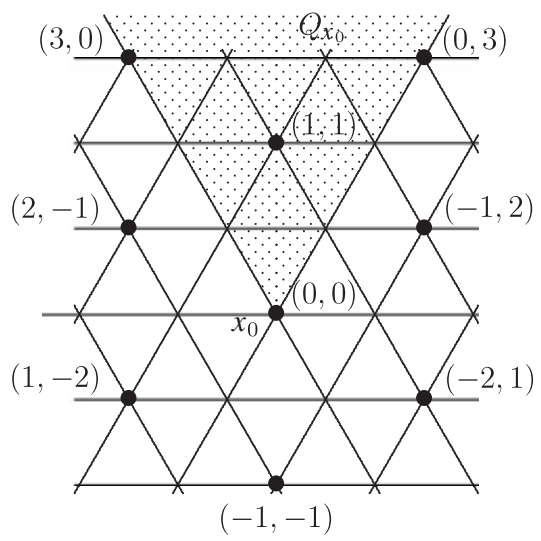

FIGURE 1. Vertices in an apartment.

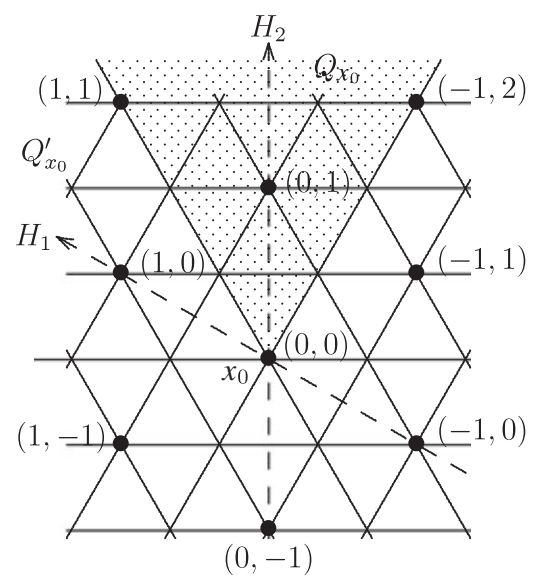

FIGURE 2. Vertices of type zero.

As we did for buildings of type $\tilde{G}_{2}$ and $\tilde{B}_{2}$, to each chamber $c$ of $\mathcal{A}$ we can assign a triple of integer coordinates $\left(k, m_{0}, n_{0}\right)$ with respect to $Q_{x_{0}}$, where $\left(m_{0}, n_{0}\right) \in \mathbb{Z}^{2}$ are the coordinates in $\mathcal{U}_{0}$ with respect to $Q_{x_{0}}$ of the vertex $x$ of type 0 lying on $c$ and $k \in\{1, \ldots, 6\}$ characterizes (among the chambers of $\mathcal{A}$ sharing the vertex $x$ ) the position of $c$ with respect to the sector $Q_{x} \sim Q_{x_{0}}$. Figure 3 exhibits the chosen numbering.

\section{Poisson kernel}

On the fundamental apartment $\mathbb{A}$, we consider, for any vertex $X \in \mathbb{U}_{0}$, the coordinates $\left(m_{0}, n_{0}\right)$ introduced in Section 2 , with respect to $\mathbb{Q}_{0}$. For every triple $\mathbf{a}=$ $\left(a_{1}, a_{2}, a_{3}\right)$ of complex numbers such that $a_{1} a_{2} a_{3}=1$, we define $\psi_{\mathbf{a}}$, the multiplicative 


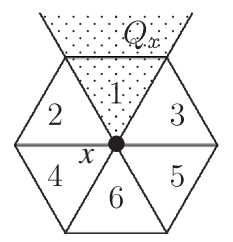

Figure 3. Coordinates of chambers.

function on $\mathbb{U}_{0}$ associated with a, by

$$
\psi_{\mathbf{a}}\left(X_{m_{0}, n_{0}}\right)=a_{1}^{m_{0}} a_{3}^{-n_{0}} \quad \forall\left(m_{0}, n_{0}\right) \in \mathbb{Z}^{2} .
$$

This function can be viewed as the restriction to $\mathbb{U}_{0}$ of the multiplicative function $\phi_{\mathbf{b}}$ on $\mathbb{U}$, associated with the triple $\mathbf{b}=\left(b_{1}, b_{2}, b_{3}\right)$, where

$$
a_{1}=b_{1} b_{2}^{-1}, \quad a_{2}=b_{2} b_{3}^{-1}, \quad a_{3}=b_{3} b_{1}^{-1} .
$$

Actually, if a vertex $X$ of $\mathbb{U}_{0}$ has coordinates $(m, n)$ in $\mathbb{U}$ and $\left(m_{0}, n_{0}\right)$ in $\mathbb{U}_{0}$, then (3.1) implies that

$$
\phi_{\mathbf{b}}(X)=b_{1}^{m} b_{3}^{-n}=\left(b_{1} b_{2}^{-1}\right)^{m_{0}}\left(b_{1}^{-1} b_{3}\right)^{-n_{0}}=a_{1}^{m_{0}} a_{3}^{-n_{0}}=\psi_{\mathbf{a}}(X) .
$$

Let $\Omega$ denote, as usual, the maximal boundary of the building. For every $\omega \in \Omega$ and $x_{0} \in \mathcal{U}_{0}$, the retraction $r_{\omega}^{x_{0}}$ with respect to $\omega$ (having initial point $x_{0}$ ) of the building on its fundamental apartment is defined as in [6]. According to [6, Definition 2.7], for any $x_{0} \in \mathcal{U}_{0}$ and any multiplicative function $\psi_{\mathbf{a}}$ on $\mathbb{U}_{0}$, the Poisson kernel with initial point $x_{0}$ associated with $\psi_{\mathbf{a}}$ is defined by

$$
P_{\mathbf{a}}^{x_{0}}(x, \omega)=\psi_{\mathbf{a}}\left(r_{\omega}^{x_{0}}\right) \quad \forall x \in \mathcal{U}_{0} \forall \omega \in \Omega
$$

We simply write $P(x, \omega)$ instead of $P_{\mathbf{a}}^{x_{0}}(x, \omega)$, whenever there is no ambiguity. The Poisson kernel depends on the initial point, by [6, Lemma 2.8, Equation (1)]. We remark that this definition agrees with [7, Definition 3.2.1] and [8, Definition 2.3.1] when we set $a_{1}=\alpha$ and $a_{3}^{-1}=\beta$.

Let $r_{c}$ be the retraction of $\Delta$ on $\mathbb{A}$, with respect to a chamber $c$, as in [6, Definition 2.3]. The retraction, $\tilde{f}_{c}$, of a function $f$, with respect to $c$, is defined by

$$
\tilde{f}_{c}(X)=\frac{1}{\left|r_{c}^{-1}(X)\right|} \sum_{x \in r_{c}^{-1}(X)} f(x) \quad \forall X \in \mathbb{U}_{0} .
$$

The method described in [8, Section 3] allow us to determine the retraction of the Poisson kernel with respect to any chamber. We define $6 \times 6$ matrices $\mathbb{M}_{0}, \mathbb{M}_{1}$ and $\mathbb{M}_{2}$ 
as follows:

$$
\begin{aligned}
\mathbb{M}_{0}=\mathbb{M}_{0}(\mathbf{a}) & =\left(\begin{array}{ccccccc}
q-1 & 0 & 0 & 0 & 0 & q a_{3} \\
0 & q-1 & 0 & 0 & q a_{1}^{-1} & 0 \\
0 & 0 & q-1 & q a_{1} a_{3} & 0 & 0 \\
0 & 0 & a_{1}^{-1} a_{3}^{-1} & 0 & 0 & 0 \\
0 & a_{1} & 0 & 0 & 0 & 0 \\
a_{3}^{-1} & 0 & 0 & 0 & 0 & 0
\end{array}\right) \\
\mathbb{M}_{1} & =\left(\begin{array}{ccccccc}
0 & 0 & 1 & 0 & 0 & 0 \\
0 & 0 & 0 & 1 & 0 & 0 \\
q & 0 & q-1 & 0 & 0 & 0 \\
0 & q & 0 & q-1 & 0 & 0 \\
0 & 0 & 0 & 0 & 0 & 1 \\
0 & 0 & 0 & 0 & q & q-1
\end{array}\right) \\
\mathbb{M}_{2} & =\left(\begin{array}{cccccc}
0 & 1 & 0 & 0 & 0 & 0 \\
q & q-1 & 0 & 0 & 0 & 0 \\
0 & 0 & 0 & 0 & 1 & 0 \\
0 & 0 & 0 & 0 & 0 & 1 \\
0 & 0 & q & 0 & q-1 & 0 \\
0 & 0 & 0 & q & 0 & q-1
\end{array}\right)
\end{aligned}
$$

Fix $\omega \in \Omega$ and a chamber $c$. If we assume that $r_{c}(c)=C=r_{\omega}^{x_{0}}(c)$ and consider the coordinates defined in Section 2 on the chamber $C$, then the following theorem holds.

Theorem 3.1. Let $\left(k, m_{0}, n_{0}\right)$ be the coordinates of the chamber $C$. For every $X \in \mathbb{U}_{0}$, let $D$ be the chamber containing $X$ in a minimal gallery connecting $C$ to $X$. Let $\pi=\left(i_{1}, \ldots, i_{l}\right)$ be the type of the gallery $[C, D]$, and let $\mathbb{M}_{\pi}=\mathbb{M}_{i_{l}} \cdots \mathbb{M}_{i_{1}}$. Then

$$
\tilde{P}(X, \omega)=\tilde{P}_{c}(X, \omega)=\frac{1}{\left|r_{c}^{-1}(D)\right|} a_{1}^{m_{0}} a_{3}^{-n_{0}} \mathbb{V}_{0} \mathbb{M}_{\pi} \mathbf{e}_{k},
$$

where $\mathbb{V}_{0}$ is the $1 \times 6$ matrix with all entries 1 , and $\mathbf{e}_{k}$ is the $6 \times 1$ matrix such that $e_{h, k}=\delta_{h k}$, when $h, k=1, \ldots, 6$.

Remark 3.2. As in [7, Remark 3.2.3], if $r_{c}(c)=C=r_{\omega}^{x_{0}}(c)$, then for every $X \in \mathbb{U}_{0}$,

$$
\sum_{x \in r_{c}^{-1}(X)} F\left(r_{\omega}^{x_{0}}(x)\right)=\sum_{X^{\prime} \in \tilde{R}(X)} h\left(X^{\prime}\right) F\left(X^{\prime}\right)
$$

where

$$
\begin{gathered}
\tilde{R}(X)=\left\{X^{\prime}=r_{\omega}^{x_{0}}(x): x \in r_{c}^{-1}(X)\right\}, \\
h\left(X^{\prime}\right)=\left|\left\{x \in r_{c}^{-1}(X): r_{\omega}^{x_{0}}(x)=X^{\prime}\right\}\right| \quad \forall X^{\prime} \in \tilde{R}(X) .
\end{gathered}
$$

Since $\tilde{R}(X)$ and $h\left(X^{\prime}\right)$ do not depend on the function $F$, they can be evaluated by choosing $F=\psi_{\mathbf{a}}$ and then applying Theorem 3.1. 


\section{Laplace operators on the fundamental apartment $\mathbb{A}$}

Let us consider the coordinate system on $\mathbb{U}$ defined in [6]. If we define $X+Y=$ $X_{m+j, n+k}$, for $X=X_{m, n}$ and $Y=X_{j, k}$, then $\mathbb{U}$ can be seen as a $W_{0}$-invariant lattice of the Euclidean plane containing $\mathbb{U}_{0}$ as a $W_{0}$-invariant sublattice. As usual, we define, for every $Y \in \mathbb{U}$, a difference operator $t_{Y}$ (acting on the complex-valued functions on $\mathbb{U}$ ) by setting

$$
t_{Y}(F)(X)=F(X+Y) \quad \forall X \in \mathbb{U} .
$$

Thus, for every $Y \in \mathbb{U}$, the operator

$$
T_{Y}=\sum_{\sigma \in W_{0}} t_{\sigma(Y)}
$$

is a $W_{0}$-invariant difference operator on $\mathbb{U}$. We simply write $T_{Y}=T_{j, k}$ when $Y=X_{j, k}$. Since

$$
\begin{aligned}
& \left\{\sigma\left(X_{j, k}\right): \sigma \in W_{0}\right\}=\left\{X_{j, k}, X_{j+k,-k}, X_{-j, j+k}, X_{-j-k, j}, X_{k,-j-k}, X_{-k,-j}\right\} \\
& \left\{\sigma\left(X_{j, 0}\right): \sigma \in W_{0}\right\}=\left\{X_{j, 0}, X_{-j, j}, X_{0,-j}\right\} \\
& \left\{\sigma\left(X_{0, k}\right): \sigma \in W_{0}\right\}=\left\{X_{0, k}, X_{k,-k}, X_{-k, 0}\right\}
\end{aligned}
$$

whenever $j, k \in \mathbb{Z} \backslash\{0\}$, it follows that, for every complex-valued function $F$ defined on $\mathbb{U}$ and every vertex $X_{m, n}$,

$$
\begin{aligned}
T_{j, k} F\left(X_{m, n}\right)= & F\left(X_{m+j, n+k}\right)+F\left(X_{m+j+k, n-k}\right)+F\left(X_{m-j, n+j+k}\right) \\
& +F\left(X_{m-j-k, n+j}\right)+F\left(X_{m+k, n-j-k}\right)+F\left(X_{m-k, n-j}\right), \\
T_{j, 0} F\left(X_{m, n}\right)= & F\left(X_{m+j, n}\right)+F\left(X_{m-j, n+j}\right)+F\left(X_{m, n-j}\right), \\
T_{0, k} F\left(X_{m, n}\right)= & F\left(X_{m, n+k}\right)+F\left(X_{m+k, n-k}\right)+F\left(X_{m-k, n}\right) .
\end{aligned}
$$

We remark that, in every case, the set $\left\{\sigma\left(X_{j, k}\right): \sigma \in W_{0}\right\}$ contains one vertex lying on the fundamental sector $\mathbb{Q}_{0}$. Therefore, whenever $(j, k) \neq(0,0)$, there exist $\bar{j}, \bar{k} \geq 0$, such that $T_{j, k}=T_{\bar{j}, \bar{k}}$. The linear span of the operators $\left\{T_{j, k}: j, k \geq 0\right\}$ consists of all $W_{0}$-invariant difference operators on $\mathbb{U}$ with constant coefficients. It is the commutative algebra $\mathcal{H}(\mathbb{A})$ generated by the operators $T_{1,0}$ and $T_{0,1}$, the so-called Laplacians for all vertices of $\mathbb{A}$ (see [1, Proposition 2.3] or [5, Proposition 3.5], with $q=1$ ). This algebra is called the Hecke algebra on $\mathbb{U}$.

If we consider $\mathbb{U}_{0}$, then, for each $Y \in \mathbb{U}_{0}$, we still let $t_{Y}$ and $T_{Y}$ denote the restriction of the previous operators to the functions defined on $\mathbb{U}_{0}$. Moreover, we denote by $\mathcal{H}_{0}(\mathbb{A})$ the subalgebra of $\mathcal{H}(\mathbb{A})$ consisting of all $W_{0}$-invariant difference operators on $\mathbb{U}_{0}$ with constant coefficients, which is called the Hecke algebra on $\mathbb{U}_{0}$. We now assign to each vertex of $\mathbb{U}_{0}$, the coordinates defined in $\mathbb{U}$. Then, for nonnegative $j, k$, the operator $T_{j, k}$ belongs to $\mathcal{H}_{0}(\mathbb{A})$ if and only if $(j, k)=(3 l+h, h)$ where $h \geq 0$ and $3 l \geq-h$. 


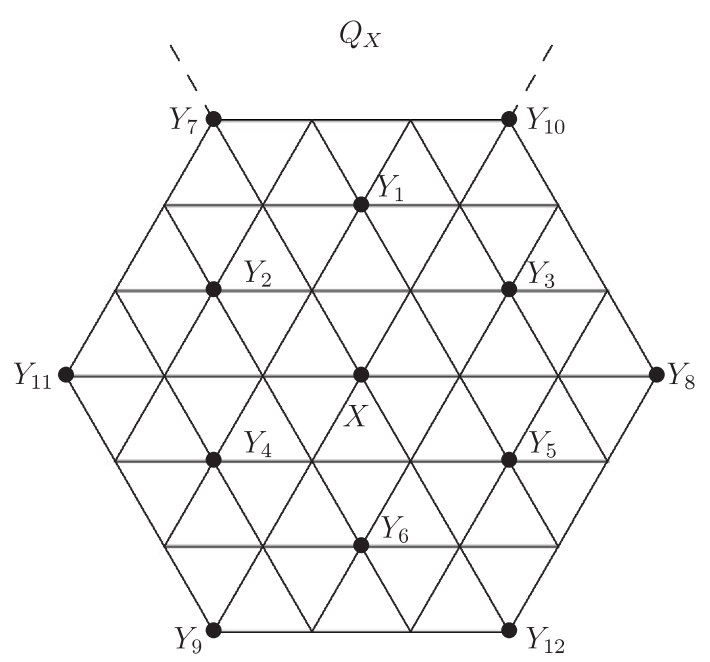

Figure 4. Vertices defining operators.

In particular, we consider, in the algebra $\mathcal{H}_{0}(\mathbb{A})$, the operators $T_{1,1}, T_{3,0}$ and $T_{0,3}$. For every function $F$ and every vertex $X=X_{m, n}$ in $\mathbb{U}_{0}$,

$$
T_{1,1} F(X)=\sum_{Y \in S_{1,1}(X)} F(Y), \quad T_{3,0} F(X)=\sum_{Y \in S_{3,0}(X)} F(Y), \quad T_{0,3} F(X)=\sum_{Y \in S_{0,3}(X)} F(Y),
$$

where

$$
\begin{aligned}
S_{1,1}(X) & =\left\{X_{m+1, n+1}, X_{m+2, n-1}, X_{m-1, n+2}, X_{m+1, n-2}, X_{m-2, n+1}, X_{m-1, n-1}\right\} \\
& =\left\{Y \in \mathbb{U}_{0}: \pi(X, Y)=(0)\right\}, \\
S_{3,0}(X) & =\left\{X_{m+3, n}, X_{m-3, n+3}, X_{m, n-3}\right\} \\
& =\left\{Y \in \mathbb{U}_{0}: \pi(X, Y)=(0,2,1,0)\right\}, \\
S_{0,3}(X) & =\left\{X_{m, n+3}, X_{m+3, n-3}, X_{m-3, n}\right\} \\
& =\left\{Y \in \mathbb{U}_{0}: \pi(X, Y)=(0,1,2,0)\right\} .
\end{aligned}
$$

See Figure 4, in which

$$
\begin{gathered}
Y_{1}=X_{m+1, n+1}, \quad Y_{2}=X_{m+2, n-1}, \quad Y_{3}=X_{m-1, n+2}, \quad Y_{4}=X_{m+1, n-2}, \\
Y_{5}=X_{m-2, n+1}, \quad Y_{6}=X_{m-1, n-1}, \quad Y_{7}=X_{m+3, n}, \quad Y_{8}=X_{m-3, n+3}, \\
Y_{9}=X_{m, n-3}, \quad Y_{10}=X_{m, n+3}, \quad Y_{11}=X_{m+3, n-3} \quad \text { and } \quad Y_{12}=X_{m-3, n} .
\end{gathered}
$$

It is easy to prove that $T_{1,1}, T_{3,0}$ and $T_{0,3}$ generate the commutative algebra $\mathcal{H}_{0}(\mathbb{A})$.

While the algebra $\mathcal{H}(\mathbb{A})$ and the corresponding algebra for a building of type $\tilde{B}_{2}$ or $\tilde{G}_{2}$ have two generators, we shall prove that the algebra $\mathcal{H}_{0}(\mathbb{A})$ requires three generators. To this end, we need some technical results. 
Lemma 4.1. Let $j, k>0$. The following formulas hold.

$$
T_{3,0} T_{j, 0}=T_{j+3,0}+c T_{j-3,3},
$$

where $c=2$ if $j=3$ and $c=1$ otherwise.

$$
T_{3,0} T_{0, k}=T_{3, k}+c T_{0, k-3},
$$

where $c=3$ if $k=3$ and $c=1$ otherwise.

$$
T_{3,0} T_{j, k}=T_{j+3, k}+c T_{j-3, k+3}+d T_{j, k-3},
$$

where $c=2$ if $j=3, d=2$ if $k=3$, and $c=d=1$ otherwise.

$$
T_{1,1} T_{j, 0}=T_{j+1,1}+c T_{j-1,2}+d T_{j-2,1}
$$

where $c=d=2$ if $j=1$ while $c=1$ and $d=2$ if $j=2$, and $c=d=1$ otherwise.

$$
T_{1,1} T_{0, k}=T_{1, k+1}+c T_{2, k-1}+d T_{1, k-2},
$$

where $c=d=2$ if $k=1$ while $c=1$ and $d=2$ if $k=2$, and $c=d=1$ otherwise.

$$
\begin{aligned}
& T_{1,1} T_{j, k}=T_{j+1, k+1}+c_{-1,2} T_{j-1, k+2}+c_{2,-1} T_{j+2, k-1} \\
& \quad \quad+c_{-2,1} T_{j-2, k+1}+c_{1,-2} T_{j+1, k-2}+c_{-1,-1} T_{j-1, k-1},
\end{aligned}
$$

where $c_{r, s}=1$ if $j+r, k+s \neq 0$ while $c_{r, s}=6$ if $j+r=k+s=0$, and $c_{r, s}=2$ otherwise.

PRoof. These formulas can be proved by direct computation.

Remark 4.2. Suppose that $(j, k)=(h+3 l, h)$ for some $h, l \in \mathbb{Z}$ such that $h, h+3 l \geq 0$. This can only occur in (4.1), (4.2), (4.4) and (4.5) for pairs $\left(j^{\prime}, k^{\prime}\right)$ with $j^{\prime}$ or $k^{\prime}$ nonnegative. In (4.3), the integers $j-3$ and $k-3$ are negative if $j=k=1$ and if $j=k=2$. In (4.6), the integers $j-2$ and $k-2$ are negative if $j=k=1$. In these exceptional cases, there are different formulas: (4.3) can be rewritten

$$
\begin{aligned}
& T_{3,0} T_{1,1}=T_{4,1}+T_{2,2}+T_{1,1}, \\
& T_{3,0} T_{2,2}=T_{5,2}+T_{1,4}+T_{1,1},
\end{aligned}
$$

and (4.6) can be rewritten

$$
T_{1,1} T_{1,1}=T_{2,2}+2 T_{0,3}+2 T_{3,0}+2 T_{1,1}+6 I \text {. }
$$

We define a partial ordering on $\mathbb{N}^{2}$ by setting

$$
(m, n) \prec\left(m^{\prime}, n^{\prime}\right)
$$


if $|(m+3 n, m)|<\left|\left(m^{\prime}+3 n^{\prime}, m^{\prime}\right)\right|$, where, as usual, $|(j, k)|$ denotes the Euclidean distance of the point $(j, k)$ from the origin. For every pair $(m, n) \in \mathbb{N}^{2}$, consider the operator $T_{1,1}^{m} T_{3,0}^{n}$. We can write

$$
T_{1,1}^{m} T_{3,0}^{n}=\sum_{h, l \in \mathbb{Z}} c_{h, l}^{m, n} T_{h+3 l, h},
$$

where all but finitely many coefficients $c_{h, l}^{m, n}$ are equal to zero when $k, l \in \mathbb{Z}, h \geq 0$ and $h+3 l \geq 0$. The following lemma gives useful information about these coefficients.

Lemma 4.3. For every $(m, n) \in \mathbb{N}^{2}$,

$$
\begin{gathered}
c_{m, n}^{m, n}=1, \\
c_{h, l}^{m, n}=0 \quad \forall(h, l) \in\left\{(h, l) \in \mathbb{N}^{2}:(h, l)>(m, n)\right\} .
\end{gathered}
$$

Proof. We assume that $m=0$ and use induction on $n$. The statements are true when $n=1$, since $T_{3,0}=T_{h+3 l, h}$ if $h=0$ and $l=n=1$. Suppose that $n>1$ and

$$
T_{3,0}^{n}=\sum_{h, l \in \mathbb{Z}} c_{h, l}^{0, n} T_{h+3 l, h},
$$

where $c_{0, n}^{0, n}=1$ and $c_{h, l}^{0, n}=0$ for all $(h, l) \in \mathbb{N}^{2}$ such that $(h, l)>(0, n)$. Then

$$
\begin{aligned}
T_{3,0}^{n+1} & =T_{3,0} \sum_{h, l} c_{h, l}^{0, n} T_{h+3 l, h}=\sum_{h, l} c_{h, l}^{0, n} T_{3,0} T_{h+3 l, h} \\
& =T_{3,0} T_{3 n, 0}+\sum_{0<l<n} c_{0, l}^{0, n} T_{3,0} T_{3 l, 0}+\sum_{h>0, h+3 l=0} c_{h, l}^{0, n} T_{3,0} T_{0, h}+\sum_{h>0, h+3 l>0} c_{h, l}^{0, n} T_{3,0} T_{h+3 l, h},
\end{aligned}
$$

and the coefficients satisfy (4.7). All the products, $T_{3,0} T_{3 n, 0}, T_{3,0} T_{3 l, 0}$ when $l<n$, $T_{3,0} T_{0, h}$ when $(h, 0) \leq(0, n)$, and $T_{3,0} T_{h+3 l, h}$ when $(h, l) \leq(0, n)$, can be calculated using Lemma 4.1. We obtain

$$
T_{3,0}^{n+1}=T_{3(n+1), 0}+\sum_{h^{\prime}, l^{\prime} \in \mathbb{Z}} c_{h^{\prime}, l^{\prime}}^{0, n+1} T_{h^{\prime}+3 l^{\prime}, h^{\prime}}
$$

where $\left(h^{\prime}, l^{\prime}\right) \leq(0, n+1)$ or $\left(h^{\prime}, l^{\prime}\right) \notin \mathbb{N}^{2}$. Hence the required statement is true when $m=0$ and $n \geq 1$.

Now, we fix $n$ and prove by induction on $m$ that the statement is true for all $m$. It is certainly true when $m=0$, as we proved before. Moreover, if we assume that

$$
T_{1,1}^{m} T_{3,0}^{n}=\sum_{h, l \in \mathbb{Z}} c_{h, l}^{m, n} T_{h+3 l, h},
$$


where $c_{m, n}^{m, n}=1$ and $c_{h, l}^{m, n}=0$ for all $(h, l) \in \mathbb{N}^{2}$ such that $(h, l)>(m, n)$, then

$$
\begin{aligned}
T_{1,1}^{m+1} T_{3,0}^{n}= & T_{1,1} \sum_{h, l} c_{h, l}^{m, n} T_{h+3 l, h}=\sum_{h, l} c_{h, l}^{m, n} T_{3,0} T_{h+3 l, h} \\
= & T_{1,1} T_{3 n, 0}+\sum_{0<l<n} c_{0, l}^{m, n} T_{1,1} T_{3 l, 0}+\sum_{h>0, h+3 l=0} c_{h, l}^{m, n} T_{1,1} T_{0, h} \\
& +\sum_{h>0, h+3 l>0} c_{h, l}^{m, n} T_{1,1} T_{h+3 l, h},
\end{aligned}
$$

where the coefficients satisfy property (4.7). Using Lemma 4.1 again, we obtain

$$
T_{1,1}^{m+1} T_{3,0}^{n}=T_{3 n+m+1, m+1}+\sum_{h^{\prime}, l^{\prime} \in \mathbb{Z}} c_{h^{\prime}, l^{\prime}}^{m+1, n} T_{h^{\prime}+3 l^{\prime}, h^{\prime}},
$$

where $\left(h^{\prime}, l^{\prime}\right) \leq(m+1, n)$ or $\left(h^{\prime}, l^{\prime}\right) \notin \mathbb{N}^{2}$.

THeOREM 4.4. The algebra $\mathcal{H}_{0}(\mathbb{A})$ cannot be generated by only two of the operators $T_{1,1}, T_{3,0}$ and $T_{0,3}$.

Proof. First we prove that the operator $T_{0,3}$ does not belong to the algebra generated by $T_{1,1}$ and $T_{3,0}$. It will then follow that $T_{3,0}$ does not belong to the algebra generated by $T_{1,1}$ and $T_{0,3}$ by symmetry.

Suppose that $T_{0,3}$ belongs to the algebra generated by $T_{1,1}$ and $T_{3,0}$. Then there are finitely many $a_{m, n}$, where $(m, n) \in \mathbb{N}^{2}$, such that

$$
T_{0,3}=\sum_{m, n} a_{m, n} T_{1,1}^{m} T_{3,0}^{n} .
$$

Therefore

$$
T_{0,3}=\sum_{m, n} a_{m, n} \sum_{h, l \in \mathbb{Z}} c_{h, l}^{m, n} T_{h+3 l, h}=\sum_{h, l \in \mathbb{Z}} b_{h, l} T_{h+3 l, h},
$$

where $b_{h, l}=\sum_{m, n} a_{m, n} c_{h, l}^{m, n}$ for all $(h, l) \in \mathbb{Z}^{2}$. On the other hand, $T_{0,3}=T_{h+3 l, h}$ when $h=3$ and $l=-1$. Therefore $b_{h, l}=0$ for all $h, l \geq 0$.

Consider the coefficients $c_{h, l}^{m, n}$, where $(m, n),(h, l) \in \mathbb{N}^{2}$. We can arrange these coefficients as a matrix, according to the partial order defined in $\mathbb{N}^{2}$. By Lemma 4.1, this matrix is lower triangular, and $c_{h, l}^{m, n}=1$ if $(h, l)=(m, n)$. This implies that the coefficients $a_{m, n}$ are uniquely determined by the coefficients $b_{h, l}$ where $h, l \geq 0$. So, in as much as $\sum_{m, n} a_{m, n} c_{h, l}^{m, n}=0$ for all $h, l \geq 0$, we must have $a_{m, n}=0$ for all $(m, n)$. This is absurd, by the definition of $a_{m, n}$.

Finally, we note that the algebra generated by $T_{3,0}$ and $T_{0,3}$ is the linear span of all operators $T_{m, n}$ with $m=3 \mu$ and $n=3 v$. Hence $T_{1,1}$ does not belong to this algebra.

Definition 4.5. Let $L_{1}=T_{1,1}, L_{2}=T_{3,0}$ and $L_{3}=T_{0,3}$. The operators $L_{1}, L_{2}$ and $L_{3}$, acting on the space of the complex-valued functions $F$ on $\mathbb{U}_{0}$, are called the Laplace operators on the type 0 vertices of the fundamental apartment $\mathbb{A}$. 
REMARK 4.6. We note that, if we assign to each vertex of $\mathbb{U}_{0}$ the coordinates $\left(m_{0}, n_{0}\right)$ defined in Section 2, then

$$
L_{1}=T_{1,0}, \quad L_{2}=T_{1,1} \quad \text { and } \quad L_{3}=T_{-1,2} .
$$

Although all the Laplace operators are required to generate the algebra $\mathcal{H}_{0}(\mathbb{A})$, the following relation connects them.

Proposition 4.7. The following formula holds:

$$
\left(L_{2}+3 L_{1}+6 I\right)\left(L_{3}+3 L_{1}+6 I\right)=\left(L_{1}+3 I\right)^{3} .
$$

Proof. We note that

$$
T_{1,0} T_{0,1}=T_{1,1}+3 I, \quad T_{1,0}^{3}=T_{3,0}+3 T_{1,1}+6 I, \quad T_{0,1}^{3}=T_{1,3}+3 T_{1,1}+6 I .
$$

Since $T_{1,0}^{3} T_{0,1}^{3}=\left(T_{1,0} T_{0,1}\right)^{3}$, the required formula holds.

Let us define operators

$$
R_{1}=L_{1}+3 I, \quad R_{2}=L_{2}+3 L_{1}+6 I, \quad R_{3}=L_{3}+3 L_{1}+6 I .
$$

The operators $R_{1}, R_{2}$ and $R_{3}$ generate the algebra $\mathcal{H}_{0}(\mathbb{A})$ and, by Proposition 4.7 , they satisfy the identity

$$
R_{2} R_{3}=R_{1}^{3} \text {. }
$$

Lemma 4.8. The commutative algebra $\mathcal{H}_{0}(\mathbb{A})$ is isomorphic to the quotient algebra

$$
\mathbb{K}=\mathbb{C}\left[X_{1}, X_{2}, X_{3}\right] /\left(X_{2} X_{3}-X_{1}^{3}\right) .
$$

Proof. Let $\iota$ be the canonical homomorphism that maps the quotient algebra $\mathbb{K}$ into $\mathcal{H}_{0}=\mathbb{C}\left[R_{1}, R_{2}, R_{3}\right]$. Obviously, $\iota$ is surjective. We prove that $\iota$ is also injective.

Let $k \in \mathbb{K}$. Then $k$ is a coset of the form

$$
k=\left[k_{0}\left(X_{2}, X_{3}\right)+k_{1}\left(X_{2}, X_{3}\right) X_{1}+k_{2}\left(X_{2}, X_{3}\right) X_{1}^{2}\right],
$$

for suitable polynomials $k_{0}, k_{1}, k_{2}$. Hence if $k \in \operatorname{ker}(\iota)$, then

$$
k_{0}\left(R_{2}, R_{3}\right)+k_{1}\left(R_{2}, R_{3}\right) R_{1}+k_{2}\left(R_{2}, R_{3}\right) R_{1}^{2}=0 .
$$

We shall prove that $k_{0}=k_{1}=k_{2}=0$. To this end, we recall (see [6]) that, for every triple $\mathbf{b}=\left(b_{1}, b_{2}, b_{3}\right)$ of complex numbers such that $b_{1} b_{2} b_{3}=1$, the multiplicative function $\phi_{\mathbf{b}}$ on $\mathbb{U}$ is a joint eigenfunction of the operators $T_{1,0}$ and $T_{0,1}$, with eigenvalues

$$
\lambda_{1}(\mathbf{b})=b_{1}+b_{2}+b_{3} \quad \text { and } \quad \lambda_{2}(\mathbf{b})=b_{1}^{-1}+b_{2}^{-1}+b_{3}^{-1} .
$$

Moreover, for every pair $\mathbf{s}=\left(s_{1}, s_{2}\right) \in \mathbb{C}^{2}$, there exists $\mathbf{b}$ such that $s_{i}=\lambda_{i}(\mathbf{b})$ when $i=1$, 2. Now $R_{1}=T_{1,0} T_{0,1}, R_{2}=T_{1,0}^{3}$, and $R_{3}=T_{0,1}^{3}$, so $\phi_{\mathbf{b}}$ is a joint eigenfunction of $R_{1}, R_{2}$ and $R_{3}$, with eigenvalues $s_{1} s_{2}, s_{1}^{3}$ and $s_{2}^{3}$ respectively. Therefore (4.8) implies that

$$
k_{0}\left(s_{1}^{3}, s_{2}^{3}\right)+k_{1}\left(s_{1}^{3}, s_{2}^{3}\right) s_{1} s_{2}+k_{2}\left(s_{1}^{3}, s_{2}^{3}\right)\left(s_{1} s_{2}\right)^{2}=0 \quad \forall s_{1}, s_{2} \in \mathbb{C},
$$

and so $k_{0}=k_{1}=k_{2}=0$. 
REMARK 4.9. The previous lemma shows that each element of $\mathcal{H}_{0}(\mathbb{A})$ can be uniquely written in the form

$$
k_{0}\left(R_{2}, R_{3}\right)+k_{1}\left(R_{2}, R_{3}\right) R_{1}+k_{2}\left(R_{2}, R_{3}\right) R_{1}^{2},
$$

for suitable polynomials $k_{0}, k_{1}$ and $k_{2}$. This means that, although the algebra $\mathcal{H}_{0}(\mathbb{A})$ requires three generators, it has dimension two.

If we define

$$
\begin{aligned}
& S_{1}^{\sharp}(X)=S_{1}(X) \cup\{X\}, \\
& S_{i}^{\sharp}(X)=S_{i}(X) \cup S_{1}(X) \cup\{X\},
\end{aligned}
$$

where $i=2,3$, then the linear operators $\Lambda_{i}^{\sharp}$, where $i=1,2,3$, given by

$$
\Lambda_{i}^{\sharp} F(X)=\sum_{Y \in S_{i}^{\sharp}(X)} h_{i}(X, Y) F(Y) \quad \forall X \in \mathbb{U}_{0},
$$

with coefficients $h_{i}(X, Y) \in \mathbb{C}$, generate the algebra $\mathcal{H}_{0}(\mathbb{A})$ if and only if $h_{i, i} \neq 0$ and

$$
h_{i}(X, Y)=h_{i, j} \quad \forall X \in \mathbb{U}_{0} \forall Y \in S_{j}(X)
$$

when $i=1,2,3$. The operators $\Lambda_{1}^{\#}, \Lambda_{2}^{\#}$ and $\Lambda_{3}^{\#}$ are called 'generalized' Laplace operators on $\mathbb{U}_{0}$.

\section{Laplace operators on $\Delta$}

For every $x \in \mathcal{U}_{0}$, we define sets

$$
\begin{aligned}
& S_{1}(x)=\left\{y \in \mathcal{U}_{0}: \pi(X, Y)=(0)\right\}, \\
& S_{2}(x)=\left\{y \in \mathcal{U}_{0}: \pi(X, Y)=(0,2,1,0)\right\}, \\
& S_{3}(x)=\left\{y \in \mathcal{U}_{0}: \pi(X, Y)=(0,1,2,0)\right\} .
\end{aligned}
$$

The cardinality of $S_{i}(x)$ does not depend on $x$. Actually, if $r_{c}$ denotes the retraction of $\Delta$ on $\mathbb{A}$ with respect to a chamber $c$, containing $x$, then

$$
S_{i}(x)=\bigcup_{Y \in S_{i}(X)} r_{c}^{-1}(Y)
$$

when $i=1,2,3$, where $X=r_{c}(x)$. This implies that

$$
\left|S_{1}(x)\right|=q(q+1)\left(q^{2}+q+1\right) \quad \text { and } \quad\left|S_{2}(x)\right|=\left|S_{3}(x)\right|=q^{4}\left(q^{2}+q+1\right) .
$$

We set $K_{i}=\left|S_{i}(x)\right|$ when $i=1,2,3$. 
Definition 5.1. The linear operators $\mathcal{L}_{1}, \mathcal{L}_{2}$ and $\mathcal{L}_{3}$, given by

$$
\mathcal{L}_{i} f(x)=K_{i}^{-1} \sum_{y \in \mathcal{S}_{i}(x)} f(y) \quad \forall x \in \mathcal{U}_{0}
$$

when $i=1,2,3$, acting on the space of complex-valued functions $f$ on $\mathcal{U}_{0}$, are called Laplace operators on the type 0 vertices of $\Delta$.

With the notation of [5, Section 1], $S_{1}(x), S_{2}(x)$ and $S_{3}(x)$ consist of all type 0 vertices $y$ having coordinates $(1,1),(3,0)$ and $(0,3)$ respectively with respect to $x$, in any sector based at $x$ containing $y$. Therefore $\mathcal{L}_{1}, \mathcal{L}_{2}$ and $\mathcal{L}_{3}$ (extended to $\mathcal{U}$ ) belong to the Hecke algebra of the building, that is, the commutative algebra $\mathcal{H}(\Delta)$ generated by the Laplacians defined in [5] for all vertices of the building, and obtained as the linear span of all averaging operators

$$
A_{m, n} f(x)=\frac{1}{K_{m, n}} \sum_{y \in S_{m, n}(x)} f(y) \quad \forall(m, n) \in \mathbb{Z}^{2},
$$

where $S_{m, n}(x)$ denotes the set of all vertices of $\mathcal{U}$ having coordinates $(m, n)$ with respect to $x \in \mathcal{U}$, and $K_{m, n}$ denotes its cardinality (see also [1]). Moreover, they generate the subalgebra $\mathcal{H}_{0}(\Delta)$ obtained as the linear span of all averaging operators $A_{m, n}$ corresponding to $(m, n)=(3 l+h, h)$ for some $l, h \in \mathbb{Z}$. This algebra is called the Hecke algebra on the type 0 vertices of the building.

An alternative proof that the algebra $\mathcal{H}_{0}(\Delta)$ is generated by the Laplace operators $\mathcal{L}_{1}, \mathcal{L}_{2}$ and $\mathcal{L}_{3}$ is given in [1, Proposition 2.5]. There, the algebra is characterized by

$$
\{A \in \mathcal{H}(\Delta): \Phi(A)=A\},
$$

for a particular algebra isomorphism $\Phi$. The same argument can be used to prove that all of the three operators are needed.

The following lemma exhibits a useful relation between the Laplace operators on the building and the Laplace operators on its fundamental apartment. See [7, Lemma 3.5.2, Remark 3.5.3] for the analogous result on a $\tilde{B}_{2}$ building.

We assign, to each vertex of $\mathbb{U}_{0}$, the coordinates $\left(m_{0}, n_{0}\right)$ defined in Section 2 . For every complex-valued function $F$ on $\mathbb{U}_{0}$, we define

$$
G\left(X_{m_{0}, n_{0}}\right)=q^{-m_{0}-2 n_{0}} F\left(X_{m_{0}, n_{0}}\right) .
$$

Lemma 5.2. Let $\omega \in \Omega$ and $x_{0} \in \mathcal{U}_{0}$. For all $x \in \mathcal{U}_{0}$, let $X=r_{\omega}^{x_{0}}(x)$. Then, for every function $F$ defined on $\mathbb{U}_{0}$,

$$
\mathcal{L}_{1} F\left(r_{\omega}^{x_{0}}(x)\right)=c_{1,1} L_{1} G(X)+c_{1,0} G(X)
$$

where

$$
c_{1,1}=q^{2} K_{1}^{-1}, \quad c_{1,0}=\left(2 q^{2}-q-1\right) K_{1}^{-1}
$$


and, when $i=2,3$,

$$
\mathcal{L}_{i} F\left(r_{\omega}^{x_{0}}(x)\right)=c_{i, i} L_{i} G(X)+c_{i, 1} L_{1} G(X)+c_{i, 0} G(X),
$$

where

$$
c_{i, i}=q^{3} K_{i}^{-1}, \quad c_{i, 1}=q^{2}(q-1) K_{i}^{-1}, \quad c_{i, 0}=q(q-1)^{2} K_{i}^{-1} .
$$

Proof. For every $x \in \mathcal{U}_{0}$, let $c$ be the base chamber of the sector $Q_{x}(\omega)$. Assume that $r_{c}(c)=C=r_{\omega}^{x_{0}}(c)$. Then

$$
\mathcal{L}_{i} F\left(r_{\omega}^{x_{0}}(x)\right)=K_{i}^{-1} \sum_{Y \in \mathcal{S}_{i}(X)}\left(\sum_{y \in r_{c}^{-1}(Y)} F\left(r_{\omega}^{x_{0}}(y)\right)\right)
$$

when $i=1,2,3$. For every $Y \in \mathcal{S}_{i}(X)$, the inner sum can be evaluated by using Remark 3.2 and Theorem 3.1. So we obtain the required identities.

Consider now the retraction, with respect to a chamber, of the Laplace operators on the type 0 vertices of $\Delta$. As in [6-8], we prove that the Laplacians retract to linear operators on $\mathbb{A}$.

Lemma 5.3. Let $c$ be a chamber. Then, when $i=1,2,3$, there exist nonnegative functions $\chi_{i}$ such that

$$
\left(\mathcal{L}_{i} f\right)_{c}(X)=\sum_{Y \in S_{i}^{\sharp}(X)} \chi_{i}(X, Y) \tilde{f}_{c}(Y) \quad \forall X \in \mathcal{U}_{0}
$$

for every function $f$ on $\mathcal{U}_{0}$. Moreover, $\chi_{i}(X, Y)>0$ if $Y \in S_{i}(X)$.

Proof. We refer the reader to [8, Proposition 4.3.1] for the proof of this lemma.

From now on, we denote by $\tilde{\mathcal{L}}_{1}, \tilde{\mathcal{L}}_{2}$ and $\tilde{\mathcal{L}}_{3}$, the linear operators on $\mathbb{A}$ obtained by retracting $\mathcal{L}_{1}, \mathcal{L}_{2}$ and $\mathcal{L}_{3}$ with respect to any chamber. That is, when $i=1,2,3$,

$$
\tilde{\mathcal{L}}_{i} F(X)=\sum_{Y \in S_{i}^{\sharp}(X)} \chi_{i}(X, Y) F(Y) \quad \forall X \in \mathbb{U}_{0} .
$$

ReMARK 5.4. Take a function $F$ on $\mathbb{U}_{0}$. For every chamber $c$, we set $f=F \cdot r_{\omega}^{x_{0}}$ and $\tilde{F}=\tilde{f}_{c}=\left(F \cdot r_{\omega}^{x_{0}}\right)_{c}$. If $\mathbb{Q}_{0}^{\vee}$ is the sector of $\mathbb{A}$ opposite to $\mathbb{Q}_{0}$, then $r_{\omega}^{x_{0}}(x)=X$ for all $X \in \mathbb{Q}^{\vee}$ and $x \in r_{c}^{-1}(X)$. Therefore

$$
\tilde{F}(X)=F(X) \quad \forall X \in \mathbb{Q}_{0}^{\vee} .
$$

With the notation of Lemma 5.2, this implies that, for all $X$ in a subsector of $\mathbb{Q}_{0}^{\vee}$,

$$
\begin{aligned}
& \tilde{\mathcal{L}}_{1}(F)(X)=\tilde{\mathcal{L}}_{1}(\tilde{F})(X)=\left(\mathcal{L}_{1} f\right)_{c}(X)=\mathcal{L}_{1}(f)(x)=\left(c_{1,1} L_{1}+c_{1,0} I\right)(G)(X), \\
& \tilde{\mathcal{L}}_{i}(F)(X)=\tilde{\mathcal{L}}_{i}(\tilde{F})(X)=\left(\mathcal{L}_{i} f\right)_{c}(X)=\mathcal{L}_{i}(f)(x)=\left(c_{i, i} L_{i}+c_{i, 1} L_{1}+c_{i, 0} I\right)(G)(X)
\end{aligned}
$$

when $i=2,3$. 


\section{Eigenfunctions of the Laplace operators on $\mathbb{A}$}

Let us consider the Laplace operators $L_{1}, L_{2}$ and $L_{3}$ on $\mathbb{U}_{0}$. As usual, if there exists a nonzero function $F$ such that $L_{i}(F)=\lambda_{i} F$ when $i=1,2,3$, then we say that $\left(\lambda_{1}, \lambda_{2}, \lambda_{3}\right)$ is a triple of eigenvalues for these operators. In this case, by Proposition 4.7 , the following identity must be satisfied:

$$
\left(\lambda_{2}+3 \lambda_{1}+6\right)\left(\lambda_{3}+3 \lambda_{1}+6\right)=\left(\lambda_{1}+3\right)^{3} .
$$

Moreover, if we set

$$
\rho_{1}=\lambda_{1}+3, \quad \rho_{2}=\lambda_{2}+3 \lambda_{1}+6, \quad \rho_{3}=\lambda_{3}+3 \lambda_{1}+6,
$$

then $\left(\rho_{1}, \rho_{2}, \rho_{3}\right)$ is a triple of eigenvalues for the operators $R_{1}, R_{2}$ and $R_{3}$, satisfying

$$
\rho_{2} \rho_{3}=\rho_{1}^{3} .
$$

Conversely, if $\left(\rho_{1}, \rho_{2}, \rho_{3}\right)$ is a triple of eigenvalues for the operators $R_{1}, R_{2}$ and $R_{3}$, then the identity (6.2) holds and there exists a unique corresponding triple $\left(\lambda_{1}, \lambda_{2}, \lambda_{3}\right)$ of eigenvalues for $L_{1}, L_{2}$ and $L_{3}$ satisfying (6.1).

The following proposition exhibits all the possible triples of eigenvalues for the operators $R_{1}, R_{2}$ and $R_{3}$.

Proposition 6.1. For every pair $\left(s_{1}, s_{2}\right)$ of complex numbers, let $\left(\rho_{1}, \rho_{2}, \rho_{3}\right)$ be the triple

$$
\rho_{1}=s_{1} s_{2}, \quad \rho_{2}=s_{1}^{3}, \quad \rho_{3}=s_{2}^{3} .
$$

Then there exists a joint eigenfunction of the operators $R_{1}, R_{2}$ and $R_{3}$ with eigenvalues $\left(\rho_{1}, \rho_{2}, \rho_{3}\right)$. Moreover, for every triple $\left(\rho_{1}, \rho_{2}, \rho_{3}\right)$ satisfying (6.2), there exists a pair $\left(s_{1}, s_{2}\right)$ such that (6.3) holds.

Proof. We write the pair of complex numbers $\left(s_{1}, s_{2}\right)$ as s. In [6], it was proved that there exists a triple $\left(b_{1}, b_{2}, b_{3}\right)$ of complex numbers, also written as $\mathbf{b}$, such that $b_{1} b_{2} b_{3}=1$ and

$$
s_{1}=s_{1}(\mathbf{b})=b_{1}+b_{2}+b_{3}, \quad s_{2}=s_{2}(\mathbf{b})=b_{1}^{-1}+b_{2}^{-1}+b_{3}^{-1} .
$$

Moreover, the multiplicative function $\phi_{\mathbf{b}}$ is a joint eigenfunction of the operators $T_{1,0}$ and $T_{0,1}$, with eigenvalues $s_{1}$ and $s_{2}$. Now $R_{1}=T_{1,0} T_{0,1}, R_{2}=T_{1,0}^{3}$ and $R_{3}=T_{0,1}^{3}$, so $\phi_{\mathbf{b}}$ is a joint eigenfunction of $R_{1}, R_{2}$ and $R_{3}$ with eigenvalues $s_{1} s_{2}, s_{1}^{3}$ and $s_{2}^{3}$ respectively.

Assume now that $\left(\rho_{1}, \rho_{2}, \rho_{3}\right)$ satisfies (6.2). If $\rho_{2} \neq 0$, it is easy to determine $s_{1}$ and $s_{2}$ such that $\rho_{2}=s_{1}^{3}$ and $\rho_{1}=s_{1} s_{2}$. Moreover, (6.2) implies that $\rho_{3}=s_{2}^{3}$. On the other hand, if $\rho_{2}=0$, then $\rho_{1}=0$, and therefore every pair $\left(0, s_{2}\right)$ where $\rho_{3}=s_{2}^{3}$ satisfies the required identities.

Remark 6.2. Proposition 6.1 implies that every triple $\left(\rho_{1}, \rho_{2}, \rho_{3}\right)$ of eigenvalues of the operators $R_{1}, R_{2}$ and $R_{3}$ can be written as $\left(\rho_{1}(\mathbf{b}), \rho_{2}(\mathbf{b}), \rho_{3}(\mathbf{b})\right)$ for some $\mathbf{b}$, 
where $\rho_{i}(\mathbf{b})=\rho_{i}\left(s_{1}(\mathbf{b}), s_{2}(\mathbf{b})\right)$ for $i=1,2,3$. For every triple $\mathbf{b}=\left(b_{1}, b_{2}, b_{3}\right)$ of complex numbers with $b_{1} b_{2} b_{3}=1$ and for every $\sigma \in W_{0}$, we denote by $\sigma(\mathbf{b})$ the triple such that

$$
\sigma \cdot \phi_{\mathbf{b}}=\phi_{\sigma(\mathbf{b})} \quad \forall \sigma \in W_{0} .
$$

Then $s_{i}(\sigma(\mathbf{b}))=s_{i}(\mathbf{b})$ for $i=1,2$; therefore $\rho_{1}, \rho_{2}$ and $\rho_{3}$ are $W_{0}$-invariant. Moreover the multiplicative functions $\phi_{\sigma(\mathbf{b})}$, restricted to $\mathbb{U}_{0}$, are joint eigenfunctions of the operators $R_{1}, R_{2}$ and $R_{3}$ with eigenvalues $\left(\rho_{1}(\mathbf{b}), \rho_{2}(\mathbf{b}), \rho_{3}(\mathbf{b})\right)$.

Moreover, for every triple $\mathbf{b}=\left(b_{1}, b_{2}, b_{3}\right)$ of complex numbers with $b_{1} b_{2} b_{3}=1$, the multiplicative functions on $\mathbb{U}$ defined by

$$
\sigma \cdot \phi_{\mathbf{b}}=\phi_{\sigma(\mathbf{b})} \quad \forall \sigma \in W_{0}
$$

and restricted to $\mathbb{U}_{0}$, are joint eigenfunctions of the operators $R_{1}, R_{2}$ and $R_{3}$ with eigenvalues, $\left(\rho_{1}(\mathbf{b}), \rho_{2}(\mathbf{b}), \rho_{3}(\mathbf{b})\right)$.

Remark 6.3. The identity (6.3) implies that, except in the case where $\rho_{1}=\rho_{2}=0$, the set of all triples of eigenvalues can be parametrized by the pair $\left(\rho_{1}, \rho_{2}\right)$ by setting $\rho_{3}=\rho_{1}^{3} / \rho_{2}$. Moreover, if $F$ is a joint eigenfunction of the operators $R_{1}$ and $R_{2}$, corresponding to eigenvalues $\left(\rho_{1}, \rho_{2}\right)$ where $\rho_{1} \rho_{2} \neq 0$, then $F$ is also an eigenfunction of $R_{3}$ with eigenvalue $\rho_{3}=\rho_{1}^{3} / \rho_{2}$.

On the other hand, if $\rho_{1}=\rho_{2}=0$, then the eigenvalue $\rho_{3}$ cannot be determined from $\rho_{1}$ and $\rho_{2}$, and there exist functions $F$ such that $R_{1}(F)=R_{2}(F)=0$ but $R_{3}(F)=\rho_{3} F$, for every $\rho_{3}$. In this case, it is easy to find eigenfunctions of $R_{1}$ and $R_{2}$ that are not eigenfunctions for $R_{3}$. Actually, if we fix distinct $h_{1}$ and $h_{2}$, then there exist $F_{1}$ and $F_{2}$ such that $R_{1}\left(F_{i}\right)=R_{2}\left(F_{i}\right)=0$ and $R_{3}\left(F_{i}\right)=h_{i} F_{i}$ when $i=1,2$. So the function $F_{1}+F_{2}$ is an eigenfunction of $R_{1}$ and $R_{2}$, but it is not an eigenfunction of $R_{3}$.

Proposition 6.1 and Remark 6.2 extend to the Laplace operators $L_{1}, L_{2}$ and $L_{3}$. For every pair $\mathbf{s}=\left(s_{1}, s_{2}\right)$ of complex numbers, let $\left(\lambda_{1}, \lambda_{2}, \lambda_{3}\right)$ be the triple satisfying the following identities:

$$
\lambda_{1}+3=s_{1} s_{2}, \quad \lambda_{2}+3 \lambda_{1}+6=s_{1}^{3}, \quad \lambda_{3}+3 \lambda_{1}+6=s_{2}^{3} .
$$

Then there exists a joint eigenfunction of the operators $L_{1}, L_{2}$ and $L_{3}$ with eigenvalues $\left(\lambda_{1}, \lambda_{2}, \lambda_{3}\right)$. In addition, for every triple $\left(\lambda_{1}, \lambda_{2}, \lambda_{3}\right)$ satisfying $(6.1)$, there exists a pair of complex numbers $\mathbf{s}=\left(s_{1}, s_{2}\right)$ such that (6.5) holds. Moreover, since $s_{i}=s_{i}(\mathbf{b})$ for a suitable triple $\mathbf{b}$, according to the identities (6.4), the same is true for $\left(\lambda_{1}, \lambda_{2}, \lambda_{3}\right)$. Finally, the $W_{0}$-invariance of $\lambda_{i}(\mathbf{b})$ where $i=1,2,3$, follows from the $W_{0}$-invariance of $s_{i}(\mathbf{b})$ where $i=1,2$.

If we assign the coordinates $\left(m_{0}, n_{0}\right)$ defined in Section 2 to each vertex of $\mathbb{U}_{0}$, and if the triples $\mathbf{a}$ and $\mathbf{b}$ are related by (3.1), then it is easy to prove that every triple of eigenvalues of the Laplacians can be written in the following way, for a 


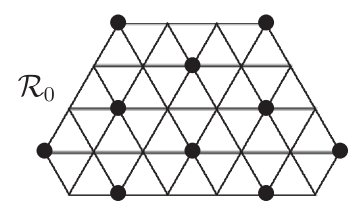

FIGURE 5. Fundamental region.

suitable $\mathbf{a}=\left(a_{1}, a_{2}, a_{3}\right)$ where $a_{1} a_{2} a_{3}=1$ :

$$
\begin{aligned}
& \lambda_{1}=\lambda_{1}(\mathbf{a})=a_{1}+a_{2}+a_{3}+a_{1}^{-1}+a_{2}^{-1}+a_{3}^{-1}, \\
& \lambda_{2}=\lambda_{2}(\mathbf{a})=a_{1} a_{3}^{-1}+a_{1}^{-1} a_{2}+a_{2}^{-1} a_{3}, \\
& \lambda_{3}=\lambda_{3}(\mathbf{a})=a_{1}^{-1} a_{3}+a_{1} a_{2}^{-1}+a_{2} a_{3}^{-1} .
\end{aligned}
$$

Moreover, for every triple $\mathbf{a}=\left(a_{1}, a_{2}, a_{3}\right)$, the multiplicative functions on $\mathbb{U}_{0}$ defined by

$$
\rho \cdot \psi_{\mathbf{a}}=\psi_{\rho(\mathbf{a})} \quad \forall \sigma \in W_{0},
$$

are joint eigenfunctions of the Laplace operators which correspond to the eigenvalues $\left(\lambda_{1}(\mathbf{a}), \lambda_{2}(\mathbf{a}), \lambda_{3}(\mathbf{a})\right)$.

Obviously, a triple $\left(\lambda_{1}, \lambda_{2}, \lambda_{3}\right)$ satisfying (6.1), or a triple $\left(\rho_{1}, \rho_{2}, \rho_{3}\right)$ satisfying (6.2), determines a homomorphism $\lambda$ of the Hecke algebra $\mathcal{H}_{0}(\mathbb{A})$ onto $\mathbb{C}$ and vice versa. According to Proposition 6.1 and Remark 6.2, for every $\lambda \in$ $\operatorname{Hom}\left(\mathcal{H}_{0}(\mathbb{A}), \mathbb{C}\right)$, there exists a, such that $\lambda=\lambda(\mathbf{a})$. Later on, we shall refer to $\lambda$ instead of $\left(\lambda_{1}, \lambda_{2}, \lambda_{3}\right)$.

For every $\lambda \in \operatorname{Hom}\left(\mathcal{H}_{0}(\mathbb{A}), \mathbb{C}\right)$, we denote by $S(\lambda)$ the joint eigenspace of the Hecke algebra $\mathcal{H}_{0}(\mathbb{A})$ corresponding to $\lambda$, that is, the joint eigenspace of the Laplace operators $L_{1}, L_{2}$ and $L_{3}$ associated with eigenvalues $\lambda_{i}=\lambda\left(L_{i}\right)$ when $i=1,2,3$,

$$
S(\lambda)=\left\{F: \mathbb{U}_{0} \rightarrow \mathbb{C}: L_{i} F=\lambda_{i} F, i=1,2,3\right\} .
$$

Proposition 6.4. For every $\lambda \in \operatorname{Hom}\left(\mathcal{H}_{0}(\mathbb{A}), \mathbb{C}\right), 6 \leq \operatorname{dim} S(\lambda) \leq 10$.

Proof. Since, for every a and $\lambda=\lambda(\mathbf{a})$, the eigenspace $S(\lambda)$ coincides with the space of the solutions of the system $\Sigma_{\psi_{\mathbf{a}}}$ of difference equations,

$$
\sum_{\sigma \in W_{0}} T_{\sigma(X)}(F)=\left(\sum_{\sigma \in W_{0}} \psi_{\mathbf{a}}(\sigma(X))\right) F \quad \forall X \in \mathbb{U}_{0},
$$

Kato [3, Proposition 1.1] states that $\operatorname{dim} S(\lambda) \geq 6$. Consider the region $\mathcal{R}_{0}$ on $\mathbb{A}$, pictured in Figure 5.

This region contains 10 vertices of type 0 . We can prove that every $F \in S(\lambda)$ is uniquely determined by its values on the type 0 vertices of $\mathcal{R}_{0}$, or of any other region of $\mathbb{A}$ obtained by applying any $w \in W$ to $\mathcal{R}_{0}$. See [8, Proposition 4.4.2] for more details. 
Let $\lambda_{0}$ be the homomorphism such that

$$
\lambda_{0}\left(L_{1}\right)=-3, \quad \lambda_{0}\left(L_{2}\right)=\lambda_{0}\left(L_{3}\right)=3 .
$$

Let $1, \alpha$ and $\alpha^{2}$ be the solutions of the equation $Z^{3}-1=0$. If we consider the triples $\alpha_{1}=(\alpha, \alpha, \alpha)$ and $\alpha_{2}=\left(\alpha^{2}, \alpha^{2}, \alpha^{2}\right)$, then it is easy to verify that $\lambda_{0}\left(L_{i}\right)=\lambda_{i}\left(\alpha_{1}\right)=$ $\lambda_{i}\left(\boldsymbol{\alpha}_{2}\right)$ for $i=1,2,3$. Actually,

$$
\lambda_{1}\left(\alpha_{1}\right)=\lambda_{1}\left(\alpha_{2}\right)=3\left(\alpha+\alpha^{2}\right)=-3 \quad \text { and } \quad \lambda_{i}\left(\alpha_{1}\right)=\lambda_{i}\left(\alpha_{2}\right)=3 \alpha^{3}=3, \quad i=2,3 .
$$

Proposition 6.5. If $\lambda \neq \lambda_{0}$, then $\operatorname{dim} S(\lambda)=6$.

Proof. Let $\lambda \in \operatorname{Hom}\left(\mathcal{H}_{0}(\mathbb{A}), \mathbb{C}\right) \backslash\left\{\lambda_{0}\right\}$. We prove that there exists $\lambda^{\prime} \in \operatorname{Hom}(\mathcal{H}(\mathbb{A}), \mathbb{C})$ such that

$$
\left(\lambda^{\prime}\right)^{3}\left(T_{1,0}\right)=\lambda\left(R_{2}\right), \quad\left(\lambda^{\prime}\right)^{3}\left(T_{0,1}\right)=\lambda\left(R_{3}\right), \quad \lambda^{\prime}\left(T_{1,0}\right) \lambda^{\prime}\left(T_{0,1}\right)=\lambda\left(R_{1}\right) .
$$

Actually, if $\lambda \neq \lambda_{0}$, then $\lambda\left(R_{2}\right) \neq 0$ or $\lambda\left(R_{3}\right) \neq 0$. We treat these two cases separately. If $\lambda\left(R_{2}\right) \neq 0$, then we define $\lambda^{\prime}\left(T_{1,0}\right)=\left(\lambda\left(R_{2}\right)\right)^{1 / 3}$ and $\lambda^{\prime}\left(T_{0,1}\right)=\lambda\left(R_{1}\right)\left(\lambda\left(R_{2}\right)\right)^{-1 / 3}$. If $\lambda\left(R_{3}\right) \neq 0$, then we define $\lambda^{\prime}\left(T_{0,1}\right)=\left(\lambda\left(R_{3}\right)\right)^{1 / 3}$ and $\lambda^{\prime}\left(T_{1,0}\right)=\lambda\left(R_{1}\right)\left(\lambda\left(R_{3}\right)\right)^{-1 / 3}$. In both cases, $\lambda^{\prime}$ satisfies the required identities.

Now, let $F \in S(\lambda)$. We prove that $F$ can be uniquely extended to a function $F^{\prime}$ on $S\left(\lambda^{\prime}\right)$. Here, $S\left(\lambda^{\prime}\right)$ denotes the joint eigenspace of the full algebra $\mathcal{H}(\mathbb{A})$. First, we define $F^{\prime}(X)=F(X)$ for all vertices of type 0 and then we define $F^{\prime}$ on all other vertices, again distinguishing two cases.

On the one hand, if $\lambda^{\prime}\left(T_{1,0}\right) \neq 0$, then we define $F^{\prime}(Y)=\lambda^{\prime}\left(T_{1,0}\right)^{-1} T_{1,0}\left(F^{\prime}\right)(Y)$ for every type 1 vertex $Y$, and $F^{\prime}(Z)=\lambda^{\prime}\left(T_{1,0}\right)^{-1} T_{1,0}\left(F^{\prime}\right)(Z)$ for every type 2 vertex $Z$. On the other hand, if $\lambda^{\prime}\left(T_{0,1}\right) \neq 0$, we simply replace $T_{0,1}$ by $T_{1,0}$ in the above definitions. We first define $F^{\prime}(Z)$ for vertices $Z$ of type 2 and then define $F^{\prime}(Y)$ for vertices $Y$ of type 1 .

Obviously, the function $F^{\prime}$ so defined belongs to $S\left(\lambda^{\prime}\right)$. Since $\operatorname{dim} S\left(\lambda^{\prime}\right)=6$, as proved in [6], the existence of the injection from $S(\lambda)$ into $S\left(\lambda^{\prime}\right)$ defined above implies that $\operatorname{dim} S\left(\boldsymbol{\lambda}^{\prime}\right) \leq 6$. Proposition 6.4 allows us to finish the proof.

Proposition 6.6. If $\lambda=\lambda_{0}$, then $\operatorname{dim} S(\lambda)=10$.

Proof. Let $\lambda=\lambda_{0}$. In this case, we exhibit 10 linearly independent functions on $\mathbb{U}_{0}$ which are eigenfunctions of the Laplacians corresponding to eigenvalues $\lambda_{1}=-3$ and $\lambda_{2}=\lambda_{2}=3$.

We assign the coordinate $\left(m_{0}, n_{0}\right)$ defined in Section 2 to every vertex of $\mathbb{U}_{0}$. We consider the multiplicative functions on $\mathbb{U}_{0}$ corresponding to the triples $\mathbf{a}_{1}$ and $\mathbf{a}_{2}$ respectively:

$$
\psi_{\boldsymbol{\alpha}_{1}}\left(X_{m_{0}, n_{0}}\right)=\alpha^{m_{0}} \alpha^{2 n_{0}}, \quad \psi_{\boldsymbol{\alpha}_{2}}\left(X_{m_{0}, n_{0}}\right)=\alpha^{2 m_{0}} \alpha^{n_{0}} .
$$

We also consider the functions $\chi_{i} \psi_{\alpha_{1}}$ and $\chi_{i} \psi_{\alpha_{2}}$, where $i=1,2,3,4$, given by

$$
\begin{aligned}
& \chi_{1}\left(X_{m_{0}, n_{0}}\right)=m_{0}, \quad \chi_{2}\left(X_{m_{0}, n_{0}}\right)=n_{0}, \\
& \chi_{3}\left(X_{m_{0}, n_{0}}\right)=m_{0}\left(m_{0}+2 n_{0}\right), \quad \chi_{4}\left(X_{m_{0}, n_{0}}\right)=n_{0}\left(2 m_{0}+n_{0}\right) .
\end{aligned}
$$


It is easy to verify that the ten functions defined above are linearly independent. Moreover,

$$
\begin{aligned}
L_{1}\left(\psi_{\boldsymbol{\alpha}_{1}}\right) & =L_{1}\left(\psi_{\boldsymbol{\alpha}_{2}}\right)=3\left(\omega+\omega^{2}\right)=-3, \\
L_{i}\left(\psi_{\alpha_{1}}\right) & =L_{i}\left(\psi_{\boldsymbol{\alpha}_{2}}\right)=3 \omega^{3}=3,
\end{aligned}
$$

when $i=2,3$. By direct computation, we can prove that the functions $\chi_{i} \psi_{\alpha_{1}}$ and $\chi_{i} \psi_{\alpha_{2}}$, where $i=1,2,3,4$, also belong to $S\left(\lambda_{0}\right)$.

Remark 6.7. In [3, Proposition 1.1], Kato states that the stabilizer in $W_{0}$ of the multiplicative functions $\psi_{\mathbf{a}}$ is a reflection group if and only if $\operatorname{dim} S(\lambda(\mathbf{a}))$ is equal to $\left|W_{0}\right|$. Therefore, Proposition 6.6 implies that the stabilizer in $W_{0}$ of the multiplicative functions $\psi_{\mathbf{a}_{1}}$ and $\psi_{\mathbf{a}_{2}}$ on $\mathbb{U}_{0}$ is not a reflection group.

Propositions 6.4, 6.5 and 6.6 easily extend to the triple of generalized Laplace operators $\Lambda_{1}^{\sharp}, \Lambda_{2}^{\sharp}$ and $\Lambda_{3}^{\sharp}$ defined in Section 4 , and also to $\tilde{\mathcal{L}}_{1}, \tilde{\mathcal{L}}_{2}$ and $\tilde{\mathcal{L}}_{3}$.

\section{Eigenfunctions of the Laplace operators on $\Delta$}

The following lemma shows how the eigenvalues of the Laplace operators on the building are related to the eigenvalues of the Laplace operators on the fundamental apartment.

Lemma 7.1. For every triple $\left(\lambda_{1}, \lambda_{2}, \lambda_{3}\right)$ of complex numbers, let

$$
\begin{aligned}
\zeta_{1} & =c_{1,1} \lambda_{1}+c_{1,0}, \\
\zeta_{i} & =c_{i, i} \lambda_{i}+c_{i, 1} \lambda_{1}+c_{i, 0}, \quad i=2,3,
\end{aligned}
$$

where the $c_{i, j}$ are defined as in Lemma 5.2. Then $\left(\lambda_{1}, \lambda_{2}, \lambda_{3}\right)$ satisfies (6.1) if and only if $\left(\zeta_{1}, \zeta_{2}, \zeta_{3}\right)$ satisfies the following identity:

$$
\begin{aligned}
{\left[q^{2} \zeta_{2}\right.} & \left.+q(q+1)(2 q+1) \zeta_{1}+(q+1)\right]\left[q^{2} \zeta_{3}+q(q+1)(2 q+1) \zeta_{1}+(q+1)\right] \\
& =q^{5}\left(q^{2}+q+1\right)\left[q(q+1) \zeta_{1}+1\right]^{3}
\end{aligned}
$$

Moreover, $\left(\zeta_{1}, \zeta_{2}, \zeta_{3}\right)$ is a triple of eigenvalues of the operators $\mathcal{L}_{1}, \mathcal{L}_{2}$ and $\mathcal{L}_{3}$ if and only if $\left(\lambda_{1}, \lambda_{2}, \lambda_{3}\right)$ is a triple of eigenvalues of the operators $L_{1}, L_{2}$ and $L_{3}$.

Proof. For every function $G$ on $\mathbb{U}_{0}$, let $F$ be the function defined by

$$
F\left(X_{m_{0}, n_{0}}\right)=q^{m_{0}+2 n_{0}} G\left(X_{m_{0}, n_{0}}\right),
$$

for every $\left(m_{0}, n_{0}\right)$ in $\mathbb{Z}^{2}$. If $G$ is a joint eigenfunction of the Laplace operators $L_{1}, L_{2}$ and $L_{3}$, corresponding to eigenvalues $\left(\lambda_{1}, \lambda_{2}, \lambda_{3}\right)$, it follows, by Lemma 5.2, that, for every $\omega \in \Omega$, the function $F \cdot r_{\omega}^{x_{0}}$ is a joint eigenfunction of the Laplace operators $\mathcal{L}_{1}$, $\mathcal{L}_{2}$ and $\mathcal{L}_{3}$, corresponding to eigenvalues $\left(\zeta_{1}, \zeta_{2}, \zeta_{3}\right)$, which are related to $\left(\lambda_{1}, \lambda_{2}, \lambda_{3}\right)$ by the identities $(7.1)$. 
Lemma 7.1 allows us to extend, to the case of one type of vertices, a result which holds for the Laplace operators on all vertices of the building.

Proposition 7.2. Let $\mathbf{a}=\left(a_{1}, a_{2}, a_{3}\right)$ be a triple of complex numbers such that $a_{1} a_{2} a_{3}=$ 1 and let $\psi_{\mathbf{a}}$ be the corresponding multiplicative function on $\mathbb{U}_{0}$. For every $\omega \in \Omega$ and $x_{0} \in \mathcal{U}_{0}$, the function $P_{\mathbf{a}}^{x_{0}}(\cdot, \omega)=\psi_{\mathbf{a}} \cdot r_{\omega}^{x_{0}}$ is a joint eigenfunction of the operators $\mathcal{L}_{1}$, $\mathcal{L}_{2}$ and $\mathcal{L}_{3}$, associated with the eigenvalues $\left(\zeta_{1}(\mathbf{a}), \zeta_{2}(\mathbf{a}), \zeta_{3}(\mathbf{a})\right)$, which are given by

$$
\begin{aligned}
& \zeta_{1}(\mathbf{a})=d_{1,1}\left(a_{3}^{-1}+q a_{1}+q a_{2}+q^{3} a_{1}^{-1}+q^{3} a_{2}^{-1}+q^{4} a_{3}\right)+d_{1,0}, \\
& \zeta_{2}(\mathbf{a})=d_{2,2}\left(a_{1} a_{3}^{-1}+q^{3} a_{1}^{-1} a_{2}+q^{6} a_{2}^{-1} a_{3}\right) \\
& \quad+d_{2,1}\left(a_{3}^{-1}+q a_{1}+q a_{2}+q^{3} a_{1}^{-1}+q^{3} a_{2}^{-1}+q^{4} a_{3}\right)+d_{2,0}, \\
& \zeta_{3}(\mathbf{a})=d_{3,3}\left(a_{2} a_{3}^{-1}+q^{3} a_{1} a_{2}^{-1}+q^{6} a_{1}^{-1} a_{3}\right) \\
& \quad+d_{3,1}\left(a_{3}^{-1}+q a_{1}+q a_{2}+q^{3} a_{1}^{-1}+q^{3} a_{2}^{-1}+q^{4} a_{3}\right)+d_{3,0},
\end{aligned}
$$

where $d_{i, 1}=q^{-2} c_{i, 1}, d_{i, 0}=c_{i, 0}$, for $i=1,2,3$, and $d_{i, i}=q^{-3} c_{i, i}$, for $i=2,3$.

Proof. Let

$$
F\left(X_{m_{0}, n_{0}}\right)=\psi_{\mathbf{a}}\left(X_{m_{0}, n_{0}}\right) \text { and } G\left(X_{m_{0}, n_{0}}\right)=q^{-m_{0}-2 n_{0}} \psi_{\mathbf{a}}\left(X_{m_{0}, n_{0}}\right),
$$

for every $\left(m_{0}, n_{0}\right)$ in $\mathbb{Z}^{2}$. Then $G=\psi_{\xi}$, where

$$
\boldsymbol{\xi}=\left(\xi_{1}, \xi_{2}, \xi_{3}\right)=\left(q^{-1} a_{1}, q^{-1} a_{2}, q^{2} a_{3}\right) .
$$

This implies, by (7.1), that $G$ is a joint eigenfunction of $L_{1}, L_{2}$ and $L_{3}$, associated with the eigenvalues

$$
\begin{aligned}
& \lambda_{1}=\lambda_{1}(\boldsymbol{\xi})=\xi_{1}+\xi_{2}+\xi_{3}+\xi_{1}^{-1}+\xi_{2}^{-1}+\xi_{3}^{-1}, \\
& \lambda_{2}=\lambda_{2}(\boldsymbol{\xi})=\xi_{1} \xi_{3}^{-1}+\xi_{1}^{-1} \xi_{2}+\xi_{2}^{-1} \xi_{3}, \\
& \lambda_{3}=\lambda_{3}(\boldsymbol{\xi})=\xi_{2} \xi_{3}^{-1}+\xi_{1} \xi_{2}^{-1}+\xi_{1}^{-1} \xi_{3} .
\end{aligned}
$$

Hence Lemma 7.1 implies that $P_{\mathbf{a}}^{x_{0}}(\cdot, \omega)=\psi_{\mathbf{a}} \cdot r_{\omega}^{x_{0}}$ is a joint eigenfunction of the operators $\mathcal{L}_{1}, \mathcal{L}_{2}$ and $\mathcal{L}_{3}$, associated with the eigenvalues $\zeta_{i}=\zeta_{i}(\mathbf{a})$ given by (7.2).

We note that Proposition 7.2 can be proved by using the same argument as in $[8$, Proposition 4.2.1].

For every a, we define

$$
\boldsymbol{\xi}(\mathbf{a})=\left(q^{-1} a_{1}, q^{-1} a_{2}, q^{2} a_{3}\right) .
$$

Lemma 7.1 and Proposition 7.2 imply that $\left(\zeta_{1}(\mathbf{a}), \zeta_{2}(\mathbf{a}), \zeta_{3}(\mathbf{a})\right)$ is a triple of eigenvalues for the Laplace operators $\mathcal{L}_{1}, \mathcal{L}_{2}$ and $\mathcal{L}_{3}$ on $\mathcal{U}_{0}$ if and only if

$$
\left(\lambda_{1}(\boldsymbol{\xi}(\mathbf{a})), \lambda_{1}(\boldsymbol{\xi}(\mathbf{a})), \lambda_{1}(\boldsymbol{\xi}(\mathbf{a}))\right)
$$

is a triple of eigenvalues for the Laplace operators $L_{1}, L_{2}$ and $L_{3}$ on $\mathcal{H}_{0}(\mathbb{A})$.

Since, for every $\left(\lambda_{1}, \lambda_{2}, \lambda_{3}\right)$, there exists a triple $\boldsymbol{\xi}$ such that $\lambda_{i}=\lambda_{i}(\boldsymbol{\xi})$ for $i=$ $1,2,3$, every triple of eigenvalues $\left(\zeta_{1}, \zeta_{2}, \zeta_{3}\right)$ of the Laplace operators on $\mathcal{U}_{0}$ can be parametrized by a triple $\mathbf{a}=\left(a_{1}, a_{2}, a_{3}\right)$, according to (7.2). 
Definition 7.3. The triples $\mathbf{a}$ and $\mathbf{a}^{\prime}$ are said to be equivalent, and we write $\mathbf{a} \sim \mathbf{a}^{\prime}$ if $\zeta_{i}(\mathbf{a})=\zeta_{i}\left(\mathbf{a}^{\prime}\right)$ for $i=1,2,3$.

Consider, for every a, the triple $\boldsymbol{\xi}=\boldsymbol{\xi}(\mathbf{a})$. For every $\sigma \in W_{0}$, we set $\mathbf{a}^{\prime}=\sigma(\mathbf{a})$ if $\psi_{\xi^{\prime}}=\sigma \psi_{\xi}$.

Corollary 7.4. The triples $\mathbf{a}$ and $\mathbf{a}^{\prime}$ are equivalent if and only if $\mathbf{a}^{\prime}=\sigma(\mathbf{a})$, for some $\sigma \in W_{0}$. Moreover, for every $\mathbf{a}$, the functions

$$
P_{\sigma(\mathbf{a})}^{x_{0}}(\cdot, \omega) \quad \forall \sigma \in W_{0},
$$

are joint eigenfunctions of the Laplace operators on $\mathcal{U}_{0}$, corresponding to the eigenvalues $\left(\zeta_{1}, \zeta_{2}, \zeta_{3}\right)$.

For every triple $\left(\zeta_{1}, \zeta_{2}, \zeta_{3}\right)$ we define sets

$$
\mathcal{S}\left(\zeta_{1}, \zeta_{2}, \zeta_{3}\right)=\left\{f: \mathcal{U}_{0} \rightarrow \mathbb{C}: \mathcal{L}_{i} f=\zeta_{i} f, i=1,2,3\right\}
$$

and

$$
\tilde{\mathcal{S}}\left(\zeta_{1}, \zeta_{2}, \zeta_{3}\right)=\left\{F: \mathbb{U}_{0} \rightarrow \mathbb{C}: \tilde{\mathcal{L}}_{i} F=\zeta_{i} F, i=1,2,3\right\} .
$$

For every function $f \in \mathcal{S}\left(\zeta_{1}, \zeta_{2}, \zeta_{3}\right)$, the retraction $\tilde{f}_{c}$ of $f$, with respect to any chamber $c$, belongs to $\tilde{\mathcal{S}}\left(\zeta_{1}, \zeta_{2}, \zeta_{3}\right)$. Moreover, as in the case of all vertices, if $F \in \tilde{\mathcal{S}}\left(\zeta_{1}, \zeta_{2}, \zeta_{3}\right)$, then the function $f$ such that

$$
f(x)=F(X) \quad \forall x \in r_{c}^{-1}(X),
$$

belongs to $\mathcal{S}\left(\zeta_{1}, \zeta_{2}, \zeta_{3}\right)$.

Actually, it is easy to prove that for such a function, $\mathcal{L}_{i} f$ is constant on the $r_{c}$-fibers, for $i=1,2,3$. Then, we can deduce from the identities $\left(\mathcal{L}_{i} f\right)_{c}(X)=$ $\zeta_{i} \tilde{f}_{c}(X)$, for $i=1,2,3$, that $\mathcal{L}_{i} f(x)=\zeta_{i} f(x)$, for $i=1,2,3$. We shall prove that there is a bijection between $\tilde{\mathcal{S}}\left(\zeta_{1}, \zeta_{2}, \zeta_{3}\right)$ and $S\left(\lambda_{1}, \lambda_{2}, \lambda_{3}\right)$, if the triples $\left(\zeta_{1}, \zeta_{2}, \zeta_{3}\right)$ and $\left(\lambda_{1}, \lambda_{2}, \lambda_{3}\right)$ are related by $(7.1)$.

Proposition 7.5. Let $\left(\zeta_{1}, \zeta_{2}, \zeta_{3}\right)$ and $\left(\lambda_{1}, \lambda_{2}, \lambda_{3}\right)$ be related by (7.1). Then the eigenspaces $\tilde{\mathcal{S}}\left(\zeta_{1}, \zeta_{2}, \zeta_{3}\right)$ and $S\left(\lambda_{1}, \lambda_{2}, \lambda_{3}\right)$ are isomorphic.

Proof. For every function $F$, we consider the function $G$ defined in Lemma 5.2 and, for some $c$, the function $\tilde{F}=\left(F \cdot r_{\omega}^{x_{0}}\right)_{c}$. We know that $G$ belongs to $S\left(\lambda_{1}, \lambda_{2}, \lambda_{3}\right)$ if and only if $F \cdot r_{\omega}^{x_{0}}$ belongs to $\mathcal{S}\left(\zeta_{1}, \zeta_{2}, \zeta_{3}\right)$. Therefore, if $G$ belongs to $S\left(\lambda_{1}, \lambda_{2}, \lambda_{3}\right)$, then $\tilde{F}$ belongs to $\tilde{\mathcal{S}}\left(\zeta_{1}, \zeta_{2}, \zeta_{3}\right)$.

As we observed in Remark 5.4,

$$
\tilde{F}(X)=F(X) \quad \forall X \in \mathbb{Q}^{\vee} .
$$

We choose the fundamental region $\mathcal{R}_{0}$ in such a way that it lies in $\mathbb{Q}^{\vee}$, at a big enough distance from its walls. Since each function in $S\left(\lambda_{1}, \lambda_{2}, \lambda_{3}\right)$, as well as each function in $\tilde{\mathcal{S}}\left(\zeta_{1}, \zeta_{2}, \zeta_{3}\right)$, is uniquely determined by its values on the vertices of $\mathcal{R}_{0}$, we conclude that the map $G \rightarrow \tilde{F}$ is a bijection from $S\left(\lambda_{1}, \lambda_{2}, \lambda_{3}\right)$ onto $\tilde{\mathcal{S}}\left(\zeta_{1}, \zeta_{2}, \zeta_{3}\right)$. 
As an evident consequence of Proposition 7.5, we have the following result.

Corollary 7.6. Let $\left(\zeta_{1}, \zeta_{2}, \zeta_{3}\right)$ and $\left(\lambda_{1}, \lambda_{2}, \lambda_{3}\right)$ be related by formula (7.1). If $\lambda_{1}=-3$ and $\lambda_{2}=\lambda_{3}=3$, then $\operatorname{dim} \tilde{\mathcal{S}}\left(\zeta_{1}, \zeta_{2}, \zeta_{3}\right)=10$. Otherwise, $\operatorname{dim} \tilde{\mathcal{S}}\left(\zeta_{1}, \zeta_{2}, \zeta_{3}\right)=6$.

ReMARK 7.7. A direct computation shows that $\left(\lambda_{1}, \lambda_{2}, \lambda_{3}\right)=(-3,3,3)$ if and only if

$$
\left(\zeta_{1}, \zeta_{2}, \zeta_{3}\right)=\left(-q^{-1}(q+1)^{-1}, q^{-3}, q^{-3}\right) .
$$

Let $\mathbf{a}_{1}=\left(q \alpha, q \alpha, q^{-2} \alpha\right)$ and $\mathbf{a}_{2}=\left(q \alpha^{2}, q \alpha^{2}, q^{-2} \alpha^{2}\right)$. Since $\boldsymbol{\xi}\left(\mathbf{a}_{i}\right)=\boldsymbol{\alpha}_{i}$, for $i=1,2$, using the notation of Section 6 , it is easy to see that the triples $\mathbf{a}_{1}$ and $\mathbf{a}_{2}$ are equivalent, as $\zeta_{i}\left(\mathbf{a}_{1}\right)=\zeta_{i}\left(\mathbf{a}_{2}\right)$, for $i=1,2,3$. Later on, we shall simply write

$$
\zeta_{i}^{0}=\zeta_{i}\left(\mathbf{a}_{1}\right)=\zeta_{i}\left(\mathbf{a}_{2}\right), \quad i=1,2,3 .
$$

\section{Bijectivity of the Poisson transform}

Following the notation of [6], we denote by $H(\Omega)$ the linear space of all locally constant functions on $\Omega$, and by $H^{\prime}(\Omega)$ its dual (consisting of all finitely additive measures defined on the algebra generated by the open sets of $\Omega$ ). Thus, for every triple a, the function $P_{\mathbf{a}}^{x_{0}}(x, \cdot)$ belongs to $H(\Omega)$ for every $x_{0}, x \in \mathcal{U}_{0}$.

For every triple a and $x_{0} \in \mathcal{U}_{0}$, the Poisson transform (of parameter a and initial point $x_{0}$ ) of any $v \in H^{\prime}(\Omega)$ is defined, as in [6], by

$$
\mathcal{P}_{\mathbf{a}}^{x_{0}} v(x)=\int_{\Omega} P_{\mathbf{a}}^{x_{0}}(x, \omega) d v .
$$

For ease of notation, when a is fixed, we simply denote this function by $\mathcal{P}^{x_{0}} v$. As a direct consequence of Proposition 7.2, the function $\mathcal{P}_{\mathbf{a}}^{x_{0}} v$ belongs to the eigenspace $\mathcal{S}\left(\zeta_{1}, \zeta_{2}, \zeta_{3}\right)$, where $\zeta_{i}=\zeta_{i}(\mathbf{a})$, for $i=1,2,3$.

In order to prove the bijectivity of the Poisson transform between $H^{\prime}(\Omega)$ and $\mathcal{S}\left(\zeta_{1}, \zeta_{2}, \zeta_{3}\right)$, applying the machinery used in [6-8], we investigate whether the eigenspace $\tilde{\mathcal{S}}\left(\zeta_{1}, \zeta_{2}, \zeta_{3}\right)$ has a basis consisting of functions obtained by retracting, with respect to a chamber, the Poisson kernel (of parameter a) for a suitable choice of boundary points.

Fix a chamber $c_{0}$ such that $x_{0} \in c_{0}$, and denote by $\tilde{P}(\cdot, \omega)$, the retraction with respect to the chamber $c_{0}$ of the Poisson kernel $P_{\mathbf{a}}^{x_{0}}(\cdot, \omega)$. The following definition is the same as that given in [6-8].

Definition 8.1. Let $k \in \mathbb{Z}^{+}$. We define the set $\Omega_{k}=\Omega_{k}\left(c_{0}\right)$ to be the set of all boundary points $\omega$ such that the base chamber of the sector $Q_{x_{0}}(\omega)$ has coordinates $(k, 0,0)$ with respect to the sector based at $c_{0}$ in any apartment containing both sectors.

The maximal boundary $\Omega$ splits into the disjoint union of the sets $\Omega_{1}, \ldots, \Omega_{6}$. Moreover, $\tilde{P}(\cdot, \omega)=\tilde{P}\left(\cdot, \omega^{\prime}\right)$, if $\omega, \omega^{\prime}$ belong to the same $\Omega_{k}$. 


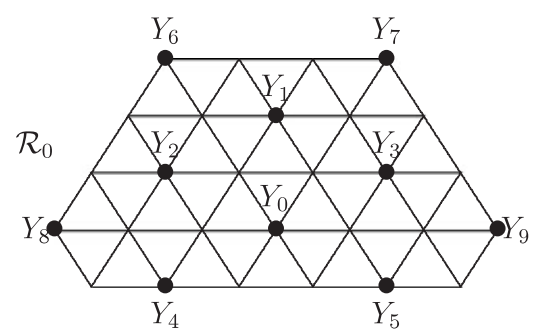

FiguRE 6. Vertices of a fundamental region.

Proposition 8.2. Let $\mathbf{a}=\mathbf{a}_{j}$ when $j=1,2$, and

$$
\zeta_{i}^{0}=\zeta_{i}\left(\mathbf{a}_{1}\right)=\zeta_{i}\left(\mathbf{a}_{2}\right), \quad i=1,2,3 .
$$

The functions $\tilde{P}\left(\cdot, \omega_{1}\right), \ldots, \tilde{P}\left(\cdot, \omega_{6}\right)$ generate a space which is properly contained in the eigenspace $\tilde{\mathcal{S}}\left(\zeta_{1}^{0}, \zeta_{2}^{0}, \zeta_{3}^{0}\right)$.

Proof. If we choose a point $\omega_{k}$ in $\Omega_{k}$, for each $k$, then we have, at most, six linearly independent functions $\tilde{P}\left(\cdot, \omega_{1}\right), \ldots, \tilde{P}\left(\cdot, \omega_{6}\right)$ lying in the eigenspace $\mathcal{S}\left(\zeta_{1}^{0}, \zeta_{2}^{0}, \zeta_{3}^{0}\right)$. On the other hand,

$$
\operatorname{dim} \mathcal{S}\left(\zeta_{1}^{0}, \zeta_{2}^{0}, \zeta_{3}^{0}\right)=\operatorname{dim} S\left(\lambda_{0}\right)=10,
$$

by Proposition 6.6. Hence, the functions $\tilde{P}\left(\cdot, \omega_{k}\right)$, for $k=1, \ldots, 6$, cannot generate the eigenspace $\mathcal{S}\left(\zeta_{1}^{0}, \zeta_{2}^{0}, \zeta_{3}^{0}\right)$.

From now on, we assume that $\left(\zeta_{1}, \zeta_{2}, \zeta_{3}\right) \neq\left(\zeta_{1}^{0}, \zeta_{2}^{0}, \zeta_{3}^{0}\right)$ or, equivalently, $\mathbf{a} \neq \mathbf{a}_{1}$ and $\mathbf{a} \neq \mathbf{a}_{2}$. In order to investigate the linear independence of the functions $\tilde{P}\left(\cdot, \omega_{1}\right), \ldots, \tilde{P}\left(\cdot, \omega_{6}\right)$, we fix a region $\mathcal{R}_{0}$ in $\mathbb{A}$ as in Figure 5 , and denote its vertices of type 0 by $Y_{1}, \ldots, Y_{10}$ (see Figure 6 ).

Moreover, we assume that $r_{\omega}^{x_{0}}$ maps $Q_{x_{0}}(\omega)$ onto the sector $\mathbb{Q}_{k}$, based at the chamber $C_{k}$ of coordinates $(k, 0,0)$ (with respect to $\mathbb{Q}_{0}$ ). Then, we construct the $10 \times 6$ matrix $\mathbb{P}=\left(P_{j, k}\right)$, where $P_{j, k}=\tilde{P}\left(Y_{j}, \omega_{k}\right)$, for every $j, k$. The entries of this matrix are determined by the following proposition.

Proposition 8.3. For every $j=1, \ldots, 10$ and $k=1, \ldots, 6$,

$$
\begin{aligned}
& \tilde{P}\left(Y_{j}, \omega_{k}\right)=\frac{1}{\left|r_{c_{0}}^{-1}\left(Y_{j}\right)\right|} \mathbb{V}_{0} \mathbb{M}_{\pi_{j}} e_{k} \quad \text { for } k \neq 4,5, \\
& \tilde{P}\left(Y_{j}, \omega_{4}\right)=\frac{1}{\left|r_{c_{0}}^{-1}\left(Y_{j}\right)\right|} \mathbb{V}_{0} \mathbb{M}_{\pi_{j}} e_{5}, \\
& \tilde{P}\left(Y_{j}, \omega_{5}\right)=\frac{1}{\left|r_{c_{0}}^{-1}\left(Y_{j}\right)\right|} \mathbb{V}_{0} \mathbb{M}_{\pi_{j}} e_{4},
\end{aligned}
$$

if $\pi_{j}$ is the type of a minimal gallery connecting $C_{0}$ to $Y_{j}$. 
Proof. If $k \neq 4,5$, then the chamber $C_{0}$ has coordinates $(k, 0,0)$ with respect to $\mathbb{Q}_{k}$. Moreover, $C_{0}$ has coordinates $(5,0,0)$ with respect to $\mathbb{Q}_{4}$ and coordinates $(4,0,0)$ with respect to $\mathbb{Q}_{5}$. Applying Theorem 3.1, we complete the proof.

The following definition extends [7, Definition 3.6.3] and [8, Definition 4.5.5].

Definition 8.4. Suppose that $\mathbf{a} \neq \mathbf{a}_{j}$ when $j=1,2$. The triple a is called singular if the characteristic of the matrix $\mathbb{P}$ is less than six.

In the following propositions, we exhibit the triples different from $\mathbf{a}_{i}$, for $i=1,2$, which are singular. For each of them, we prove that there exists an equivalent triple which is not singular.

Proposition 8.5. Let $\mathbf{a} \neq \mathbf{a}_{j}$, when $j=1,2$; if $\mathbf{a}=\left(a_{1}, a_{2}, a_{3}\right)$, then $\mathbf{a}$ is singular in the following cases:

$$
a_{1}=1 \quad \text { or } \quad a_{2}=1 \quad \text { or } \quad a_{3}=1 / q .
$$

Proof. We consider the $10 \times 6$ matrix $\mathbb{P}=\left(P_{j k}\right)$, where $P_{j k}=\tilde{P}\left(X_{j}, \omega_{k}\right)$. It contains 210 submatrices of order six, whose entries are polynomials in the variables $q, a_{1}, a_{2}, a_{3}$, with $a_{1} a_{2} a_{3}=1$. We checked, exhaustively, by using the mathematical software 'Mathematica', that all the determinants are equal to zero, if and only if a satisfies at least one of the equalities in (8.1).

Proposition 8.6. For every singular triple $\mathbf{a}$, there exists an equivalent triple $\mathbf{a}^{\prime}$ which is nonsingular.

Proof. Setting $a_{1}=q \xi_{1}, a_{2}=q \xi_{2}$ and $a_{3}=q^{-2} \xi_{3}$, then $\left(\xi_{1}, \xi_{2}, \xi_{3}\right)$ is singular if and only if at least one of the following relations is satisfied:

$$
\xi_{1}=1 / q, \quad \xi_{2}=1 / q, \quad \xi_{3}=q .
$$

Let us write, for ease of notation, $\xi=\xi_{1}$ and $\eta=\xi_{3}^{-1}$. Let

$$
\mathcal{F}=\{(\xi, \eta): 1 \leq|\eta| \leq|\xi|\}
$$

Replacing (if necessary) the pair $(\xi, \eta)$ by a pair corresponding to an equivalent triple, we may assume that $(\xi, \eta) \in \mathcal{F}$. In this set, none of the conditions (8.2) may be satisfied. So the proposition is proved.

As in $[7,8]$ we have the following theorem.

Theorem 8.7. Let $\zeta_{i}=\zeta_{i}(\mathbf{a})$, for $i=1,2,3$.

(1) For every $\mathbf{a}$, the Poisson transform $\mathcal{P}_{\mathbf{a}}^{x_{0}}$ is injective.

(2) If $\zeta_{i} \neq \zeta_{i}^{0}$, for $i=1,2,3$, then, for every $f \in \mathcal{S}\left(\zeta_{1}, \zeta_{2}, \zeta_{3}\right)$, there exists a unique $v \in H^{\prime}(\Omega)$ such that $f=\mathcal{P}_{\mathbf{a}} v$.

(3) If $\zeta_{i}=\zeta_{i}^{0}$, for $i=1,2,3$, then $\mathcal{P}_{\mathbf{a}}^{x_{0}}\left(H^{\prime}(\Omega)\right)$ is properly contained in $\mathcal{S}\left(\zeta_{1}^{0}, \zeta_{2}^{0}, \zeta_{3}^{0}\right)$.

Proof. Statement (3) follows from Proposition 8.2. We refer to [7] for the proof of statements (1) and (2). 


\title{
Acknowledgement
}

We would like to thank Tim Steger for his useful comments and suggestions about the content of this paper.

\section{References}

[1] D. I. Cartwright and W. Młotkowski, 'Harmonic analysis for groups acting on triangle buildings', J. Aust. Math. Soc. A 56 (1994), 345-383.

[2] S. Helgason, 'A duality for symmetric spaces with applications to group representations', Adv. Math. 5 (1970), 1-154.

[3] S. Kato, 'On eigenspaces of the Hecke algebra with respect to a good maximal compact subgroup of a $p$-adic reductive group', Math. Ann. 257 (1981), 1-7.

[4] F. Kellil and G. Rousseau, 'Opérateurs invariants sur certains immeubles affine de rang 2', Ann. Fac. Sci. Toulouse Math. (6) 16 (2007), 591-610.

[5] A. M. Mantero and A. Zappa, 'Spherical functions and spectrum of the Laplace operators on buildings of rank 2', Boll. Unione Mat. Ital. B (7) 8 (1994), 419-475.

[6] A. M. Mantero and A. Zappa, 'Eigenfunctions of the Laplace operators for a building of type $\tilde{A_{2}}$, J. Geom. Anal. 10 (2000), 339-363.

[7] A. M. Mantero and A. Zappa, 'Eigenfunctions of the Laplace operators for a building of type $\tilde{B}_{2}$, Boll. Unione Mat. Ital. Sez. B Artic. Ric. Mat. (8) 5 (2002), 163-195.

[8] A. M. Mantero and A. Zappa, 'Eigenfunctions of the Laplace operators for a building of type $\tilde{G}_{2}$, Boll. Unione Mat. Ital. (9) 2 (2009), 483-508.

[9] M. A. Ronan, Lectures on Buildings, Perspectives in Mathematics, 7 (Academic Press, London, 1989).

\section{A. M. MANTERO, D. S. A., Facoltà di Architettura, Università di Genova, Salita Sant'Agostino 37,16123 Genova, Italy \\ e-mail: mantero@dima.unige.it}

\author{
A. ZAPPA, D. I. M. A., Università di Genova, \\ Via Dodecaneso 35, 16146 Genova, Italy \\ e-mail: zappa@dima.unige.it
}

